\title{
Mobile Sensor Networks for Optimal Leak and Backflow Detection and Localization in Municipal Water Networks
}

\author{
Weijiao Gong ${ }^{\mathrm{a}}$, Mahima Agumbe Suresh ${ }^{\mathrm{a}}$, Lidia Smith ${ }^{\mathrm{a}}$, Avi Ostfeld ${ }^{\mathrm{b}}$, Radu Stoleru ${ }^{\mathrm{a}}$, Amin Rasekh $^{\mathrm{a}}$, M. \\ Katherine Banks ${ }^{\mathrm{a}}$ \\ ${ }^{a}$ Texas AEM University \\ ${ }^{b}$ Technion - Israel Institute of Technology
}

\begin{abstract}
Leak and backflow detections are essential aspects of water distribution systems (WDSs) monitoring and are commonly fulfilled using approaches that are based on static sensor networks and point measurements. Alternatively, we propose a mobile, wireless sensor network solution composed of mobile sensor nodes that travel freely inside the pipes with the water flow, and collect and transmit measurements in near-realtime (called sensors) and static access points (called beacons). This study complements the tremendous progress in mobile sensor technology. We formulate the sensor and beacon optimal placement task as a Mixed Integer Nonlinear Programming (MINLP) problem to maximize localization accuracy with budget constraint. Given the high time complexity of MINLP formulation, we propose a disjoint scheme that follows the strategy of splitting the sensor and beacon placement problems and determining the respective number of sensors and beacons by exhaustive search in linear time.
\end{abstract}

Keywords: mobile wireless sensor network, leak detection, backflow detection, source identification, localization, water distribution systems, municipal water networks

2010 MSC: 00-01, 99-00

\section{Introduction}

Drinking water supply for about $90 \%$ of U.S. population comes from nearly 170,000 public water distribution systems (WDSs) [1]. Spanning close to a million miles, the aging, buried pipeline infrastructure [2] [3] plays an important role in maintaining and preserving a clean and reliable water supply essential to industrial growth and public health. Since many main pipes in the urban infrastructure are reaching the end of their life and may easily date back to the early $20^{\text {th }}$ century, they are highly vulnerable to breaks and leaks. This has been manifested recently by an approximately 237,600 water main breaks per year in the U.S. causing a loss of nearly $\$ 2.8$ billion [4]. Breaks in water mains pose two key threats: (i) loss of water, service disruptions, and structural damages due to leakage; and (ii) public health risks due to backflow events.

Leakages: Water demand needs are getting tighter over time, especially with the recurrent, escalating droughts throughout the past decade [5].

Preprint submitted to Journal of LATEX Templates
In such stressful scenarios, water leakages are costly. A large percentage of the estimated 48.6 billion cubic meters water lost worldwide every year is due to leakages in water pipes [6]. Water leakage causes severe economic losses by means other than the loss of water too, such as ground instabilities, transportation disruptions, and problems to communication lines [7].

Backflows: A backflow is an unwanted flow of non-portable water, or other substances such as soil, pesticides, etc., into WDS pipelines. Backflow events occur when there is a reverse pressure gradient at cross-connections, and are caused by cracks, breaks, or loose connections. Such events cause illness and are a major threat to public health [17] [18]. This is evident from a U.S. EPA compilation, which states that between 1970 and 2001, 459 incidents led to an estimated 12,093 illnesses [19].

The economic and health impacts of leaks and backflows warrants the replacement of worn out systems. However, the American Water Works Association's report [20] estimates that over the next 30

November 16, 2015 
Table 1: Existing technologies that use mobile sensors for WDS leak/backflow detection

\begin{tabular}{|c|c|c|c|c|}
\hline Authors/Company & Name & Capabilities & $\begin{array}{l}\text { Sensing } \\
\text { technology }\end{array}$ & $\begin{array}{l}\text { Free-flowing or } \\
\text { line tethered }\end{array}$ \\
\hline Pure Technologies & Sahara [8] & $\begin{array}{l}\text { Detecting leaks, pockets of } \\
\text { trapped gas, and visual } \\
\text { inspection }\end{array}$ & Hydrophone; camera & Line tethered \\
\hline Pure Technologies & SmartBall [9] & $\begin{array}{l}\text { Detecting leaks, pockets of } \\
\text { trapped gas, and structural } \\
\text { defects }\end{array}$ & $\begin{array}{l}\text { Acoustic emitter and } \\
\text { receiver }\end{array}$ & Free-flowing \\
\hline Pure Technologies & PipeDiver [10] & $\begin{array}{l}\text { Detecting leaks, pockets of } \\
\text { trapped gas, and structural } \\
\text { defects }\end{array}$ & $\begin{array}{l}\text { Acoustic emitter and } \\
\text { receiver }\end{array}$ & Free-flowing \\
\hline Lai et al. & PipeProbe [11] & Mapping hidden pipeline & $\begin{array}{l}\text { Metering pressure } \\
\text { and angular velocity }\end{array}$ & Free-flowing \\
\hline Trinchero et al. & {$[12]$} & $\begin{array}{l}\text { Detecting leakage. Includes } \\
\text { wireless transmission system }\end{array}$ & Hydrophone & Free-flowing \\
\hline Chatzigeorgiou & [13] & Detecting leakage & Hydrophone & Free-flowing \\
\hline $\begin{array}{l}\text { Purdue-TAMU } \\
\text { sensor }\end{array}$ & {$[14]$} & $\begin{array}{l}\text { Measuring water quality } \\
\text { parameters; can be used for } \\
\text { backflow detection. Includes } \\
\text { energy harvest and wireless } \\
\text { transmission systems }\end{array}$ & $\begin{array}{l}\text { Ion-selective } \\
\text { electrode-based } \\
\text { biochip }\end{array}$ & Free-flowing \\
\hline MIT MRL Lab & PipeGuard [15] & $\begin{array}{l}\text { Detecting leakage. Can be } \\
\text { potentially used for backflow } \\
\text { detection }\end{array}$ & Measuring pressure & Free-flowing \\
\hline Perelman et. al. & {$[16]$} & $\begin{array}{l}\text { Monitoring water quality. } \\
\text { Uses both mobile and static } \\
\text { sensors }\end{array}$ & Water quality sensors & Free-flowing \\
\hline
\end{tabular}

years, $\$ 250$ billion may be required. For efficient replacement of the aging water pipes, the conditions of the pipeline system, such as location of breaks, cracks, loose connections are required.

\subsection{State of the art}

There exist several methods based on various operating principles for detection, localization, and pinpointing of leakages in municipal water distribution systems. Water audits based on metering and water balance calculations can be performed to quantify water losses and provide an extremely crude approximation of the location of losses. A better estimation is achieved through step-testing method whereby valves are systematically closed to subdivide the area and localize the leakage.

A comparatively more recent leak localization method is acoustic logging, which is performed using hydrophones or vibration sensors [21]. Ground penetrating radar is employed to localize the leaks by virtue of detecting underground voids caused by leakage water flow in the immediate vicinity of pipes. More accurate leakage localization, which is also referred to as leakage pinpointing, may be achieved using leak noise correlation, tracer gas, and pig-mounted acoustic techniques. Detailed description and comparison of these well-known meth- ods for detection and pinpointing of leaks may be found in [4] [22] [13]. An approach towards optimal surveillance monitoring is a method that maximizes the value of information [23] to optimally place observers that detect outbreaks as they spread. However, our leak/backflow detection model is different since the sensors are mobile, and the event does not propagate, whereas in [23], the event propagates while observers are static.

The application of inline, mobile sensors technology for leakage pinpointing has attracted a lot of attention by both researchers and practitioners during the recent years [9] [13] [11] [24] [25] [26] [27] [28] [14]. These are small monitoring devices that traverse freely with the water flow inside the pipes. The sensors can collect measurements from the environment and also process them and transmit the processed data to access points placed outside the pipelines. They have achieved popularity recently thanks to their unique abilities in collecting spatially high resolution data. Simple acoustic sensors are already being employed in real WDSs for leak detection [8] [29] and new sensors are under test and evaluation for monitoring water quality parameters in near-realtime scales [14].

Deployment of mobile sensors may happen on- 
demand by a utility manager as part of a periodic system monitoring or be triggered when the presence of a leak/backflow is suspected with the help of a static sensor system or by complaints from consumers. They have been already applied to water utilities of several cities around the world, including Dallas, Montreal, and Manila [8]. Their increasing popularity is presumably associated with their ability to pinpoint the leaks more accurately than other existing methods without causing any disruption to regular water utility service. Table 1 contains most of the existing practical solutions that use mobile sensors for leak/backflow detection in WDSs.

Although inline, mobile sensors for pinpointing leakages have been already designed and fabricated, decision support models to facilitate and enhance their operation through simulation of their movement in the pipelines network and optimization of their application is still underdeveloped. Development of such computational models is a major focus of this study.

A recent work [16] examines the deployment of mobile water quality sensors along with static water quality sensors for contamination detection. The paper demonstrates the improvement in detection rate provided by mobile sensors in conjunction with static sensors. However, the movement of the sensor is modeled to move through a deterministic path, provided the flow velocities in the pipes are known. The model use is also limited to contamination event detection and is not developed to enable source localization as well. In this paper, we consider leak/backflow detection, which requires a more stringent sensing model where the mobile sensors require to move close to the leak points to enable detection and localization. In reality, mobile sensors move randomly through the pipes with a probability distribution at each junction which has not been yet considered in existing solutions in the literature. The sensor mobility model in this paper is more general to accommodate the random movement of sensors at junctions. This way, the sensors mobility may be modeled more accurately and thus be deployed more efficiently and effectively.

Other solutions to monitor pipelines using mobile sensors are presented by the research community [30] [31] [32]. TriopusNet [31] is a solution for autonomous continuous monitoring of pipelines. The solution however assumes that the path of a sensor can be made deterministic by controlling the flow of water, which is impractical without disrupting the function of the WDS. MISE-PIPE [32] is another similar system based on magnetic induction in underground pipes. Theoretical results in pipeline monitoring using mobile sensors have also been studied. [33] performs a mathematical analysis of sensor placement and presents analysis of complex networks using graph-theoretic concepts. [34] addresses the gallery guarding problem that requires every point in the pipe to be monitored by a robot. However, these problems are specific to controlled mobile devices. The problem of ensuring $\mathrm{k}$-coverage in scenarios similar to the one we consider is presented in [35]. However, the coverage requirement is different, and mobility is ignored.

\subsection{Our Contributions}

Recent work in WDS monitoring using mobile sensors of notable interest are [24], [25] and [26], which tackle the problem of optimizing the number of sensors required to cover a region of interest in the WDS with a required degree of coverage. The papers also present a source identification technique, referred to as localization, to obtain accurate location of leaks/backflows using beacons placed outside the pipeline. We refer to the problem of optimal sensor placement as the Minimization of the Number of Sensors problem (MNS). The papers [24], [25] and [26] consider sensor and beacon placement as disjoint problems. Sensor placement to achieve a required sensing coverage is seen as requirement for localization and beacon placement problem is solved independent of sensor placement under the assumption that sensing coverage is ensured. The problem of sensor placement that obtains the optimal placement of sensors, given an upper bound on the total number of sensors has been tackled previously [27]. A simulator called FlowSim is developed and used for the evaluation of the system.

Computer models have been previously developed for the use of mobile sensors in the detection of contaminant intrusion events in WDSs [16] [36]. However, decision support models to locate the source of contamination using mobile sensors are still in an underdeveloped stage. This goal is pursued in this study through linkage of mobile sensors with ground communication stations, called beacons and minimization of contaminant intrusion source and leakage localization error subject to budget constraints. This is one of the pioneering studies to incorporate the role of ground communication stations in optimal monitoring of WDSs using mobile sensors technology. 
Table 2: Table containing the important acronyms and symbols used in this paper ${ }^{1}$

\begin{tabular}{|l|l|}
\hline Acronym/Symbol & Description \\
\hline WDS & Water Distribution Systems \\
\hline MNS & Minimization of Number of Sensors \\
\hline MINLP & Mixed Integer Non Linear Programming \\
\hline$I$ & Zone of Interest \\
\hline $\mathbf{S}$ & The sensor deployment configuration \\
\hline $\mathbf{B}$ & The beacon placement configuration \\
\hline ale & Average Leak/backflow Localization Error \\
\hline$s l e$ & System Localization Error \\
\hline$s p$ & The price of a sensor \\
\hline$b p$ & The price of a beacon \\
\hline$l e_{i j}$ & Localization error between a pair of beacons \\
\hline$e f n$ & Expected False Negative Rate \\
\hline$u b f n$ & Upper bound False Negative Rate \\
\hline MEFN & Minimization of Expected System Localization error \\
\hline$A S C$ & Average Sensing Coverage \\
\hline MASC & Maximization of Average Sensing Coverage \\
\hline MUBFN & Minimization of Upper Bound False Negative Rate \\
\hline$L B S C$ & Lower Bound Sensing Coverage \\
\hline MLBSC & Maximizing the Lower Bound Sensing Coverage \\
\hline MLALE & Minimization of Linearized Average Localization Error \\
\hline MLUBLE & Minimization of Linearized Upper Bound Localization Error \\
\hline
\end{tabular}

When a utility manager suspects that a region of the WDS contains a leak/backflow, there is a need to confirm the presence of the leak/backflow and also identify its location. We call this on-demand monitoring, where we assume that the flows in the WDS do not change for a certain duration. Efforts are underway to model the use of mobile sensors for continuous monitoring of WDSs [28], whereby sensors reside in the WDS for a long duration while detecting events and reporting them through wireless communication. This paper only deals with on-demand monitoring. A mathematical model for the joint optimization problem of sensor and beacon placement is mixed integer nonlinear programming (MINLP). Finding a solution to this MINLP problem using existing solvers is very time-consuming. Therefore we propose a disjoint solution that formulates the sensor and beacon placement problems individually and select the best combination with an exhaustive search algorithm. The contributions of this paper are:

- We present a mathematical model for the joint optimization problem of sensor and beacon placement problems minimizing the leakage/backflow localization error.
- We propose a disjoint solution with separate formulations for sensor and beacon placement problems and select the best combination in linear time.

- We demonstrate the benefit of our solution on a sample WDS from EPANET [37] and a virtual model city called Micropolis [38].

In the rest of the paper, we first present the methodology and definition of important terminology used in the paper in Section 2. We then present formulation of the problems we tackle in this paper, in Sections 3 and 4. We finally evaluate the performance of our methodology and discuss the results in Section 5.

\section{Methodology}

This section presents a thorough description of our basic models for the system, namely, definition of the terms used as well as a description of leak/backflow sensing and sensor mobility models. The important acronyms and symbols used in this paper are listed in Table 2. 


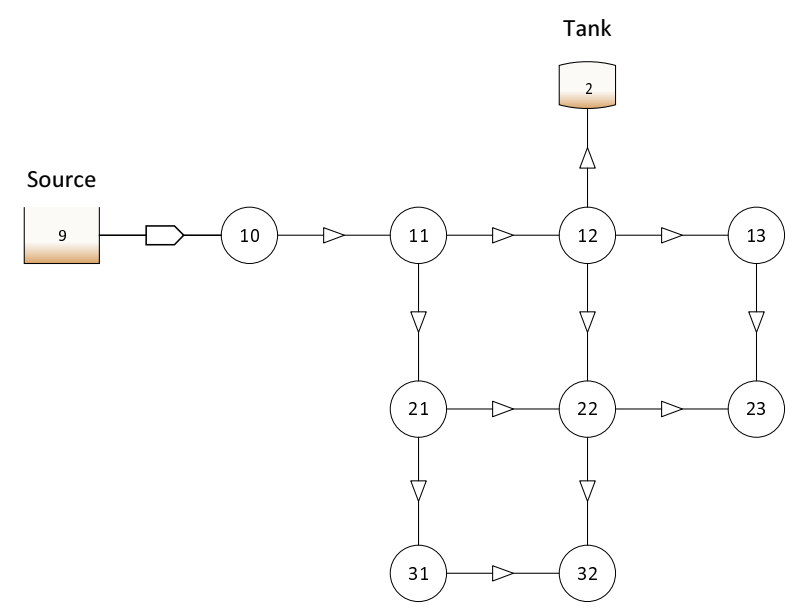

Figure 1: Net1 example water distribution network

\subsection{Zone of Interest}

A zone of interest is a certain part of the network containing a set of pipes where the utility owner/user suspects that the leak/backflow is present or where a periodic monitoring is planned to be conducted. The objective is then to detect the leak/backflow in this zone using a set of mobile sensors. The information from the sensors is collected either by wireless access points placed outside the pipes while sensors travel inside the pipes or after they are physically captured.

Zone of Interest: We define a zone of interest $I$ as a directed graph $G(V, E)$ with vertices $V$ corresponding to junctions and edges $E$ corresponding to the pipes. There are $n$ vertices in the graph, $V=\left\{v_{1}, \ldots, v_{n}\right\}$ and $m$ directed edges. An edge $e_{i j}$ is directed from initial vertex $v_{i}$ to terminal vertex $v_{j}$. The vertices are numbered in topological order so that if there is nonzero flow from $v_{i}$ to $v_{j}$ then $i<j$. Consider the Net1 example in Figure 1. An example of a zone of interest here is the set of vertices $\left\{v_{11}, v_{12}, v_{13}, v_{21}, v_{22}, v_{23}, v_{31}, v_{32}\right\}$ and the edges between them.

\subsection{Leak/Backflow Detection Model}

We assume a binary sensing model, i.e., if a sensor goes through a pipe with a leak/backflow, the leak/backflow will be detected. Therefore, for a leak/backflow to be detected, at least one sensor is required to traverse the pipe containing the leak/backflow. Typical location accuracy of Sahara leak detection technology [8], for instance, is within 1.5 feet and it can locate very small leaks (as small as $0.0005 \mathrm{gal} / \mathrm{min}$ ) as claimed by the manufacturer.
This is consistent with our model, since the length of pipes in the model city used in our evaluations, Micropolis [38], are over 3 feet. Throughout this paper, it is assumed that a leakage/backflow is detected if a sensor traverses the pipe that contains it.

\subsection{Sensor and Beacon configurations}

Sensor deployment is represented by a vector $\mathbf{S}$. In vector $\mathbf{S}=\left[s_{1}, s_{2}, \ldots, s_{r}, \ldots, s_{n}\right]$, also called the "configuration" of sensors, each entry $s_{r}$ represents the number of sensors inserted at node $v_{r}$. The time of insertion of the sensors is not of significance, as long as they are inserted before the flows in the pipes change due to varying demands. The total number of available sensors is denoted as $s$, i.e., $\sum_{i=1}^{n} s_{i}=s$.

The beacon placement is represented by a vector, $\mathbf{B}=\left\{b_{1}, b_{2}, \ldots, b_{r}, \ldots, b_{n}\right\}$, where $b_{i}$ has the value 1 if we place a beacon at junction $v_{r}$ and 0 otherwise; $b$ is the total number of beacons placed in the zone of interest, i.e., $\sum_{i=1}^{n} b_{i}=b$.

\subsection{Sensor Mobility Model}

The sensors are inserted from vertices/junctions into the WDS. The sensors move freely with water flow in pipes and their movement cannot be controlled. It is assumed that flow in pipes are known and fixed for the period of time sensors are deployed. The movement of sensors at junctions is probabilistic owing to the fluid dynamics at the junctions of the WDS. In this paper, we assume that the probability of a sensor moving into a new pipe section is dependent on the distribution of outgoing flows. The probability of moving from one vertex to another by traversing a single edge is represented in matrix $\mathbf{Q}$, where an element $q_{i j}$ of matrix $\mathbf{Q}$ is defined as the probability of taking edge $e_{i j}$ at vertex $v_{i}$ (in one transition step).

Since $G$ is a directed graph with no cycles, the matrix $\mathbf{Q}$ is an upper triangular matrix as follows:

$$
\mathbf{Q}=\left(\begin{array}{cccc}
0 & q_{12} & \ldots & q_{1 n} \\
0 & 0 & \ldots & q_{2 n} \\
\vdots & \vdots & \ddots & \vdots \\
0 & 0 & \ldots & 0
\end{array}\right)
$$

For example, in Figure 1, $q_{9,10}=1, q_{10,11}=1, \quad{ }_{319}$ $q_{11,12}=0.5640, q_{11,21}=0.4360, q_{21,22}=0.5369, \quad{ }_{320}$ $q_{21,31}=0.4631, q_{12,2}=0.7067, q_{12,22}=0.1740, \quad{ }_{321}$ $q_{12,13}=0.1193, q_{22,23}=0.6709, q_{22,32}=0.3291$. 
We model the movement of sensors through vertices as a binomial distribution. Each sensor represents a trial in the binomial experiment and the probability that a sensors travels through a certain edge is the probability of "success" for the trials. We assume that the movement of a sensor is independent of the movement of any other sensor.

We use the same definition of "traversal probability matrix" as in [27], [24], [25], and [26] to represent the probability of a sensor reaching another vertex traversing any path as:

$$
\mathbf{P}=\sum \mathbf{Q}^{k}=\mathbf{I}+\mathbf{Q}+\mathbf{Q}^{2}+\ldots
$$

An element of matrix $\mathbf{P}, p_{i j}$, is the probability of reaching vertex $v_{j}$ with a sensor starting from $v_{i}$. For the example in Figure 1, some elements of $\mathbf{P}$ are: $p_{9,10}=1, p_{9,11}=1\left(\right.$ since $\left.q_{9,10}=1, q_{10,11}=1\right)$, $p_{9,12}=0.4360\left(\right.$ since $q_{9,10}=1, q_{10,11}=1, q_{11,12}=$ 0.4360 ). When there are multiple paths between two vertices, the expression for $\mathbf{P}$ reflects the probability that a sensor follows any of the paths to reach the destination vertex. For example, in $\mathbf{P}$, $p_{11,22}=0.3322$, because $q_{11,12}=0.5640, q_{12,22}=$ 0.1740 , and $q_{11,21}=0.4360, q_{21,22}=0.5369$, i.e., $p_{11,22}=0.5640 \times 0.1740+0.4360 \times 0.5369=0.3322$. The probability that a sensor starting from vertex $v_{r}$ will visit the edge $e_{i j}$ is:

$$
p_{r, i j}^{e}=p_{r, i} \times q_{i j}
$$

The probability of the complementary event is denoted by $\beta_{r, i j}=1-p_{r, i j}^{e}$. For example, in the example illustrated in Figure 1, $p_{11 .(22,23)}^{e}=p_{11,22} \times$ $q_{22,23}=0.3322 \times 0.6709=0.2229$. As described earlier, a sensor inserted at junction $v_{11}$ has a 0.2229 probability of reaching the pipe between $v_{22}$ and $v_{23}$.

As mentioned in Section 1.2, we presume that the flows in pipes of a WDS do not change during the time at which the sensors move through the pipes, i.e., flow is not time-varying for the purpose of this study. With this assumption, the probability distribution of a sensor at a junction moving through any of its adjacent edges remains the same during the course of the sensor movement. Efforts are underway to ease this presumption for a more realistic modeling in future works [28].

\section{Probability of covering an edge:}

Consider a scenario where $s_{r}$ sensors are inserted at a single vertex $v_{r}$. The probability of none of the $s_{r}$ sensors, that travel independently, reach edge $e_{i j}$ is $\beta_{r, i j}^{s_{r}}$. Then the probability that none of the sensors reach edge $e_{i j}$ with the configuration $\mathbf{S}$ is:

$$
u_{i j}^{e}=\prod_{r=1}^{n} \beta_{r, i j}^{s_{r}}
$$

Hence, the probability that at least one sensor reaches $e_{i j}$ with the configuration $\mathbf{S}$, denoted by $P V\left(\mathbf{S}, e_{i j}\right)$, is:

$$
P V\left(\mathbf{S}, e_{i j}\right)=1-u_{i j}^{e}
$$

\subsection{Localization Error}

378

A major goal in WDS monitoring for 379 leak/backflow events is to narrow down the suspected area, as it is closely related to the scale of the problem and the time and labor it takes to pinpoint and eliminate the leaks/backflows. The design objective of our monitoring system is to minimize the scope of suspected area quantified as system localization error.

Imagine a mobile sensor inserted in vertex $v_{11}$ in Example 1 which travels to $v_{22}$ through vertex $v_{12}$. If there are beacons at vertices $v_{11}$ and $v_{22}$, and a leak/backflow in edge $e_{11,12}$, it is reported by the sensor through communication with beacons placed at vertices $v_{11}$ and $v_{22}$. Any of the four pipes between $v_{11}$ and $v_{22}$ are now suspected to contain the reported leak/backflow. But, if there was an additional beacon present at $v_{12}$, we would be able to narrow down the location of the leak/backflow to one pipe.

Beacons placed at junctions can localize the event by collecting information from the sensors passing by. The probability that a pipe contains leaks/backflows depends on its age and operation conditions. Leak probability of a pipe is denoted by $l_{i j}$ in this paper. We assume that if a utility manager is able to predict the probability of leaks, based on the age and material of the pipe, and the soil type, we provide the ability to include it in improving the monitoring performance. However, if we are not provided with the leak probability of the edges, the leak probability is assumed to be uniform.

False Negative rate:Edge localization error: We define false negative, denoted by $l e\left(\mathbf{S}, e_{i j}\right)$, as the product of its leak probability and uncovered probability, i.e. $l_{i j} \cdot u_{i j}^{e}$. For example, if we inserted only 2 sensors at junction $v_{11}$, the uncovered probability of the pipe between $v_{22}$ and $v_{23}$ is $(1-0.2229)^{2}=0.6039$. If the leak probability of 
the pipe is 0.5 , the false negative rate of the pipe is $0.6039 \times 0.5=0.3020$.

Leak/backflow localization error: For clarity, we first introduce two concepts, inner vertices and inner edges, before giving the definition of leak/backflow localization error. inner vertices is defined between two vertices $v_{i}$ and $v_{j}$ in the network, and it consists of all vertices which a sensor may pass by if it travels from $v_{i}$ to $v_{j}$, excluding $v_{i}$ and $v_{j}$ without passing by any beacon. Similarly, inner edges includes those edges which the sensor may go through during its travel without encountering any beacon. For example, when no beacons are placed, the inner vertices between $v_{11}$ and $v_{22}$ are $v_{12}$ and $v_{22}$, and inner edges are $e_{11,12}, e_{12,22}, e_{11,21}$, and $e_{21,22}$. However, if there is a beacon present at junction $v_{12}$, the inner vertex between $v_{11}$ and $v_{22}$ is $v_{22}$, and inner edges are $e_{11,21}$ and $e_{21,22}$.

Beacon localization error: The placement of beacons directly affects the localization. In this paper, when an event is localized, the utility manager is provided with a list of suspected edges where the event might be present. The leak/backflow localization errorbeacon localization error, or localization error of a leak/backflow between two adjacent beacons, say $b_{i}$ and $b_{j}$, denoted by $l e_{i j}$, is defined as the sum of localization errors of all inner edges between $v_{i}$ to $v_{j}$.

The average leak/backflowbeacon localization error, denoted by ale, is calculated by:

$$
\text { ale }=\sum_{\forall e_{k l} \in \text { edges between }\left(b_{i}, b_{j}\right)} \frac{l e_{k l}}{b p_{-n u m}}
$$

where $\left(b_{i}, b_{j}\right)$ is a pair of beacons and bp_num is the number of beacon pairs (1 if no beacon is placed). The details of the derivation of number of edges between each pair of beacons are described in Section 4.1 .

The key difference between ale and false negative rate is that ale measures the effect of both sensor and beacon placement, whereas false negative rate measures the effect of only the sensor placement. For example, if beacons are placed at all junctions, but there are no sensors deployed, the uncovered probability of all edges is high. On the other hand, the number of beacon pairs is high. However, if we did not place any beacons, but sensors are deployed such that all edges are covered with a high probability, the numerator in ale will be low, but the denominator will be 1 . However, the ale places more emphasis on the beacon placement than sen- sor placement, since the denominator is a bigger value than the numerator terms. Hence, we define a metric called system localization error.

System localization error: The metric system localization error, denoted by sle, is calculated as:

$$
s l e=A \operatorname{chSC} \cdot \text { ale }+(1-\operatorname{AchSC}) \sum_{e_{k h}} l_{k h}
$$

where $A c h S C$ is the achieved sensing coverage, i.e., $\sum_{\forall e_{i j}} P V\left(\mathbf{S}, e_{i j}\right)$. If a leak/backflow is detected, effect of sensor and beacon placement is determined to be ale. However, if the leak/backflow is not detected, the effect of sensor and beacon placement is the same as suspecting the entire WDS to contain the leak/backflow event.

For each edge, say $e_{i j}$, we introduce the indicator random variable, $\chi_{S, e_{i j}}$, that takes value 1 if leak/backflow present on edge $e_{i j}$ cannot be detected with a specific configuration of sensors $\mathbf{S}$, and value 0 otherwise. ${ }^{2}$

$$
\underline{\chi_{S, e_{i j}}}= \begin{cases}\underline{1} & \text { with probability } p=l e\left(\mathbf{S}, e_{i j}\right) \\ \underline{0} & \underline{\text { with probability } 1-p}\end{cases}
$$

The expected system loealization error, denoted by esle, is calculated by:

$$
\text { else }=\mathbb{E}\left[\sum_{\forall e_{i j}} \chi_{S, e_{i j}}\right]
$$

which is equal to the system localization error when no beacons are placed. Due to the linearity of expected value, we have $\mathbb{E}\left[\sum_{\forall e_{i j}} \chi s_{, e_{i j}}\right]=\sum_{\forall e_{i j}} \mathbb{E}\left[\chi s_{, e_{i j}}\right]$, then esle $=\sum_{\forall e_{i j}} l e\left(S, e_{i j}\right)$.

\section{Optimization Problem}

The optimization objective function is defined as the minimization of system localization error represented by Equation 2. The decision variables include the number of mobile sensors and stationary beacons used, and their deployment locations. The respective number of sensors and beacons is subject to the available budget. The constrained optimization problems is thus mathematically formulated as:

2 irrelevant 
minimize sle

subject to:

$$
\begin{gathered}
s p \cdot \sum_{r} s_{r}+b p \cdot \sum_{r} b_{r} \leq \text { cost } \\
s_{r} \in\left\{0,1, \ldots,\left\lfloor\frac{\text { cost }}{s p}\right\rfloor\right\} \\
b_{r} \in\{0,1\}
\end{gathered}
$$

where cost is the available budget for purchasing sensor and beacon devices, $s p$ is the price of a mobile sensor, $b p$ is the price of a beacon and $r=1, \ldots, n$. In all the equations in this paper, the indices range from 1 to $n$, unless specified otherwise. The first constraint represents the cost budget constraint and the last two constraints define variables $s_{r}$ to be integer and $b_{r}$ to be binary.

\section{Optimization Techniques}

The joint sensor and beacon placement optimization presented in Section 3 is a computationally intensive problem. Hence, we simplify the formulation to solve the sensor and beacon placement jointly. However, the problem continues to be nonlinear and computationally intensive. To this end, we will define two alternative formulations that separately optimize sensor and beacon placement for a given cost.

\subsection{Joint Formulation}

Minimization of sle is a complex problem, e.g., in our experiments, finding the optimal solution for a WDS with over 50 junctions in not possible even in weeks. An alternative approach used here is minimizing ale instead, which is a less complex problem and can be solved in a reasonable time.

We note here that the sle is AchSC ale + (1$A c h S C) \sum_{e_{k h}} l_{k h}$. The term AchSC in the sle formulation ensures that we place emphasis on false negatives in leak/backflow detection as well. Another notable point is that ale is also dependent both on the sensor and beacon configurations. The benefit of minimizing ale as opposed to the minimization of sle is not as harmful, as we see in the Performance Evaluation section.

The set $\left\{(i, j) \mid p_{i j}>0\right\}$ includes all nonindependent vertex pairs in graph $G$, denoted by $C N$. The set $\left\{(i, j)^{\prime} \mid p_{i j}>0\right.$, or $\left.i==j\right\}$ is denoted by $C N^{\prime}$. In order to express ale, we introduce a bi- $\quad{ }_{545}$ nary variable $c_{i j}$ to represent the presence $\left(c_{i j}=1\right) \quad{ }_{546}$ and absence $\left(c_{i j}=0\right)$ of beacons between vertices ${ }_{547}$ $v_{i}$ and $v_{j}$ including vertex $v_{j}$, defined as:

$$
\left\{\begin{array}{l}
c_{i i}=1 \\
c_{i j}=0,(i, j) \notin C N \\
c_{i j}=1-\prod_{e_{k j}}\left(1-c_{i k} \cdot\left(1-b_{j}\right)\right),(i, j) \in C N
\end{array}\right.
$$

Then, the localization error $l e_{i j}$ can be expressed $\quad{ }_{550}$ as:

$$
\begin{aligned}
l e_{i j}= & \sum_{e_{k h}}\left(c_{i k} \cdot c_{k h} \cdot \operatorname{conn}_{h j} \cdot l_{k h} \cdot u_{k h}^{e}\right) \\
& +\sum_{e_{g j}} c_{i, g} \cdot l_{g j} \cdot u_{g j}^{e}
\end{aligned}
$$

where $\operatorname{conn}_{h j}=\left(1-\prod_{e_{g j}}\left(1-c_{h g}\right)\right)$ which is 1 only ${ }_{553}$ if there exists a neighbor $v_{g}$ of vertex $v_{j}$ such that ${ }^{554}$ $c_{h g}$ is 1.

The joint formulation is a Mixed Integer NonLinear Programming $(M I N L P)$ formulation and is given as:

$$
\begin{gathered}
\text { minimize ale i.e., } \\
\operatorname{minimize} \frac{\sum_{(i, j) \in C N}\left[l e_{i j} \cdot b_{i} \cdot b_{j}\right]}{\left(\sum_{r} b_{r}\right)^{2}-\sum_{r} b_{r}}
\end{gathered}
$$

subject to the same constraints represented by Equations 4, 5, and 6 .

\subsection{Disjoint Formulation}

Although many MINLP solvers [39] [40] have been developed and can be used for solving the joint formulation, their time complexity is exponential to the problem core size. A possible idea to reduce time complexity of the problem is to split and solve the sensor and beacon placement problems separately.

Considering the computational intensity of ale minimization problem, we define two alternative formulations that separately solve the sensor and beacon placement optimization problems.

The function $l e_{i j}$ in the original formulation is a monotonically decreasing function (i.e., if $i^{\prime}>i$ and $j^{\prime}>j$, then $\left.l e_{i^{\prime} j^{\prime}} \leq l e_{i j}\right)$. A monotonic optimization problem achieve optimum solution at boundaries when constraints set is convex. The only constraint $s p \cdot \sum_{r=1}^{n} s_{r}+b p \cdot \sum_{r=1}^{n} b_{r} \leq$ cost in our formulation is convex. Therefore a separate solution will not sacrifice the optimality of the results. 


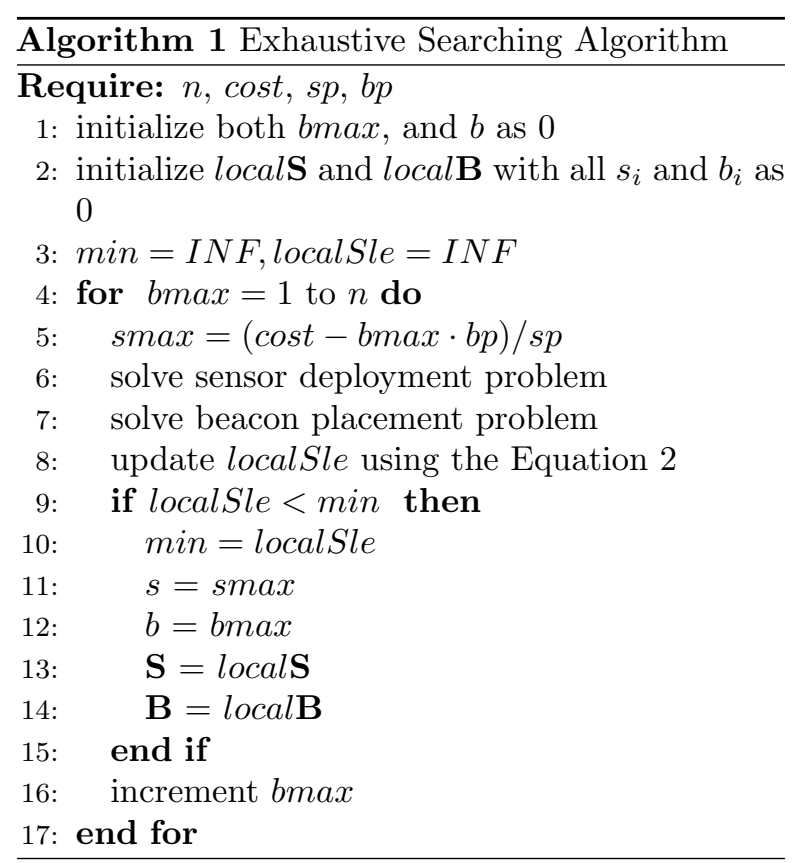

The number of cases satisfying $s p \cdot \sum_{r=1}^{n} s_{r}+b p$. $\sum_{r=1}^{n} b_{r}=$ cost is linearly related to the network size because the number of beacons is the upper bounded by the number of vertices in the network. The outline of the searching process of the disjoint method is shown in Algorithm 1. The algorithm iteratively splits the total cost among sensors and beacons starting with no beacons and adds one beacon at a time (Lines 2 and 5). For each $s$ and $b$, we solve the sensor and beacon problems separately by methods described later in this section (Lines 6 and 7). During the iterations, we record $\mathbf{S}$ and $\mathbf{B}$ that achieve the least sle (Lines 10 - 14).

The alternative disjoint formulations and algorithms for sensor deployment problem and beacon placement problem are presented in the following subsections.

\subsubsection{Sensor Deployment Problem}

Two objectives of the sensor deployment problem are to minimize expected false negative rate, i.e., efneste and to minimize upper bound false negative rate, $u b f n$. We present formulations and solutions for the two objectives this subsection.

For each edge, say $e_{i j}$, we introduce the indicator random variable, $\chi_{S, e_{i j}}$, that takes value 1 if leak/backflow present on edge $e_{i j}$ cannot be detected with a specific configuration of sensors $\mathbf{S}$, and value 0 otherwise.

$$
\chi_{S, e_{i j}}= \begin{cases}1 & \text { with probability } p=l e\left(\mathbf{S}, e_{i j}\right) \\ 0 & \text { with probability } 1-p\end{cases}
$$

The expected false negative rate, denoted by ef $n, \quad{ }_{613}$ is calculated by:

$$
e f n=\mathbb{E}\left[\sum_{\forall e_{i j}} \chi_{S, e_{i j}}\right]
$$

which is equal to the system localization error ${ }_{616}$ when no beacons are placed. Due to the linear- ${ }_{617}$ ity of expected value, we have $\mathbb{E}\left[\sum_{\forall e_{i j}} \chi_{S, e_{i j}}\right]={ }_{618}$ $\sum_{\forall e_{i j}} \mathbb{E}\left[\chi_{S, e_{i j}}\right]$, then efn $=\sum_{\forall e_{i j}} l e\left(S, e_{i j}\right)$, and ${ }_{619}$ $u b f n=\max _{\forall e_{i j}} l e\left(S, e_{i j}\right)$.

Greedy Heuristic for Minimizing ${ }_{621}$ the Expected False Negative Rate ${ }_{622}$ (MEFN)System Localization Error (MESLE): ${ }_{623}$

The problem of minimizing efneste is formulated $\quad 624$ as follows:

$$
\begin{aligned}
& \text { minimize efnesle } \text { i.e., } \\
& \operatorname{minimize} \sum_{e_{i j}}\left[l e_{i j} \cdot u_{i j}^{e}\right]
\end{aligned}
$$

subject to:

$$
\begin{aligned}
& \sum_{r=1 \ldots n} s_{r}=s \\
& s_{r} \geq 0 \quad \forall r=1 \ldots n
\end{aligned}
$$

The objective in the problem of minimizing efneste is written as:

$$
\operatorname{minimize} \sum_{e_{i j}}\left(l e_{i j} \cdot \prod_{r} \beta_{r, i j}^{s_{r}}\right)
$$

This is a convex nonlinear programming problem and we solve it using a greedy heuristic as described in Algorithm 2. Starting from an empty sensor deployment configuration (Line 1), the algorithm inserts one sensor at a time (Line 5) at a junction with the best objective function value given the sensors configuration already in place (Lines 6 - 9).

When a utility manager is only interested in detecting the presence of the leak/backflow, and not in localizing it, only the sensor deployment problem needs to be solved. In this case, we define the Average Sensing Coverage $(A S C)$ as the average probability of covering any edge, i.e., $\sum_{e_{i j}}\left[1-u_{i j}^{e}\right]$. The
632 633 634 635 636 637 638 639 640 641 642 643 644 


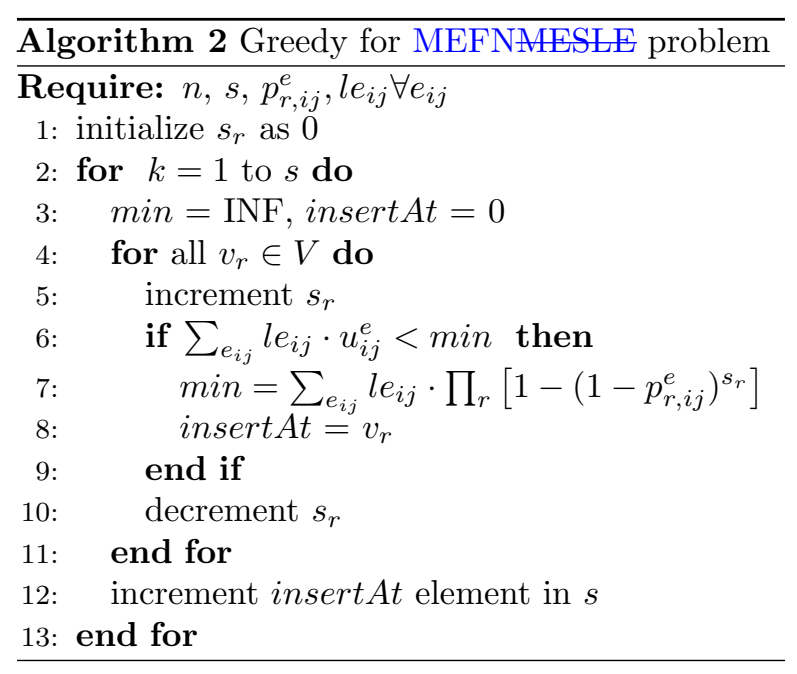

MEFNMESLE problem is then reduced to maximizing the average sensing coverage problem MASC. If the leakage/backflow probability is not considered, we solve the MASC problem using the greedy algorithm with all the leak probabilities set to to 1 , as described in [27]. The approximation ratio of greedy algorithm for MASC is $\left(1+\frac{1}{e-1}\right)$ (See Section Appendix A).

Integer Linear Programming for Minimizing the Upper Bound False Negative RateEdge Localization Error (MUBFNAUBELE):

As defined before, ubfnubete is the smallest number such that $\forall e_{i j} \in E,\left[l e\left(S, e_{i j}\right)\right] \leq u b$ fnubele. The formulation for minimizing ubfnubete is as follows:

$$
\begin{aligned}
& \text { minimize } u b f n \text {, i.e., } \\
& \text { minimize } \max _{e_{i j}}\left[\left(l e_{i j} \cdot\left(1-u_{i j}^{e}\right)\right)\right] \\
& \text { subject to: } \\
& \sum_{r=1 \ldots n} s_{i}=s \\
& s_{r} \geq 0 \quad \forall r=1 \ldots n
\end{aligned}
$$

The MUBFNMUBELE problem is a min-max problem that can be reduced to an integer linear programming problem $(I P)$ as follows:

$$
\min _{s} \max _{e_{i j}}\left[l e_{i j} \cdot u_{i j}^{e}\right]
$$

Taking the logarithm of the objective function, we get $\ln \left(l e_{i j} \cdot u_{i j}^{e}\right)=\ln \left(l e_{i j}\right)+\sum_{r} s_{r} \cdot \ln \left(\beta_{i j}\right)$, Since logarithm is a monotone increasing function and $\ln \left(l e_{i j}\right), \log \left(\beta_{i j}\right)$ are constants, the problem can be ${ }_{669}$ reduced to: subject to

$$
\begin{aligned}
& \sum_{r} \ln \left(l e_{i j}\right)+s_{r} \cdot \ln \left(\beta_{i j}\right) \leq x \\
& \sum_{r} s_{r}=s \\
& s_{r} \geq 0
\end{aligned}
$$

where $x$ is a new variable introduced to convert a ${ }_{674}$ min-max problem into a linear program.

Similar to MASC, when only sensor deployment is considered, the coverage of a pipe becomes synonymous to detection of a leak/backflow in that pipe. The Lower Bound Sensing Coverage ( $L B S C$ ) is defined as the minimum probability of covering any edge, i.e., $L B S C$ is the largest number such that $\forall e_{i j} \in E,\left[1-u_{i j}^{e}\right] \geq L B S C$. The problem of MUBFNMUBELE is then transformed into maximizing the lower bound of covering any edge (MLBSC), as described in [27].

\subsubsection{Beacon Placement Problem}

Once the sensor deployment problem is solved, the joint optimization problem is reduced to the beacon placement problem. We can further reduce the problem to a linear programming problem by linearizing the equations for $c$ and $l e_{i j}$ in Equation 7 and 8 , respectively, as follows:

$$
\left\{\begin{aligned}
c_{i i} & =1 \\
c_{i j} & =0,(i, j) \notin C N \\
c_{i j} & \geq c_{i k}-b_{j},(i, j) \in C N \\
c_{i j} & \leq 1-b_{j},(i, j) \in C N
\end{aligned}\right.
$$

The linearized $c_{r h}$ is equal to 1 when there is no ${ }_{693}$ beacon along the path from vertex $r$ to vertex $h$. 694 Its value is uncertain otherwise.

The localization error $l e_{i j}$ is linearized based on the AMGM inequality:

$$
\begin{aligned}
l e_{i j}= & \sum_{e_{k h}}^{n}\left(\frac{c_{i k}+c_{i h}+\operatorname{conn}_{k j}}{3}\right) \cdot l e_{k h} \\
& +\sum_{g} c_{i g} \cdot l e_{g j}^{e}
\end{aligned}
$$

where $\operatorname{conn}_{k j}=\left(\sum_{e_{g j}} c_{k g}\right) / n$.
686 
We define the linearized ale minimization, de- 71699 5.1. Applications noted by MLALE, as:

$$
\operatorname{minimize} \sum_{(i, j) \in C N}\left[\frac{l e_{i j}}{b \max ^{2}-b \max }\right]
$$

and define the linearized uble minimization, denoted by MLUBLE, as:

$$
\operatorname{minimize} \max _{(i, j) \in C N}\left[\frac{l e_{i j}}{b \max ^{2}-b \max }\right]
$$

The two separate solutions we propose are two different combinations of sensor placement and beacon placement formulations. The former, greedy, combines MEFNAESLE and MLALE and the latter, linear, integrates MUBFNMUBELE and MLUBLE.

Table 3: Optimization problems used in this paper

\begin{tabular}{|l|l|l|}
\hline Method & Objective & Constraints \\
\hline joint & minimizing ale & Budget \\
\hline MEFN & minimizing efn & Budget \\
\hline MUBFN & minimizing ubfn & Budget \\
\hline MLALE & minimizing ale & Budget \\
\hline mLUBLE & $\begin{array}{l}\text { minimizing linearized } \\
\text { ufn } n\end{array}$ & Budget \\
\hline greedy & $\begin{array}{l}\text { Algorithm 1 with } \\
\text { MEFN and MLALE }\end{array}$ & Budget \\
\hline linear & $\begin{array}{l}\text { Algorithm 1 with } \\
\text { MUBFN and MLU- }\end{array}$ & Budget \\
\hline BLESC & Maximizing $A S C$ & Sensor budget \\
\hline MLBSC & Maximizing $L B S C$ & Sensor budget \\
\hline MNS & $\begin{array}{l}\text { Minimizing number of } \\
\text { sensors }\end{array}$ & LBSC \\
\hline
\end{tabular}

All the optimization problems used in this paper are summarized in Table 3.

\section{Performance evaluation}

In this section, we evaluate the performance of our proposed solutions on the small example network NET1 shown in Figure 1 and three distinct zones of a large WDS belonging to Micropolis [38], a virtual city, as illustrated in Figures 2 and 3 .
Our simulations are performed using Flowsim [24], a simulator specially designed for mobile wireless sensor networks in WDSs. It is based on flows determined by EPANET [37].

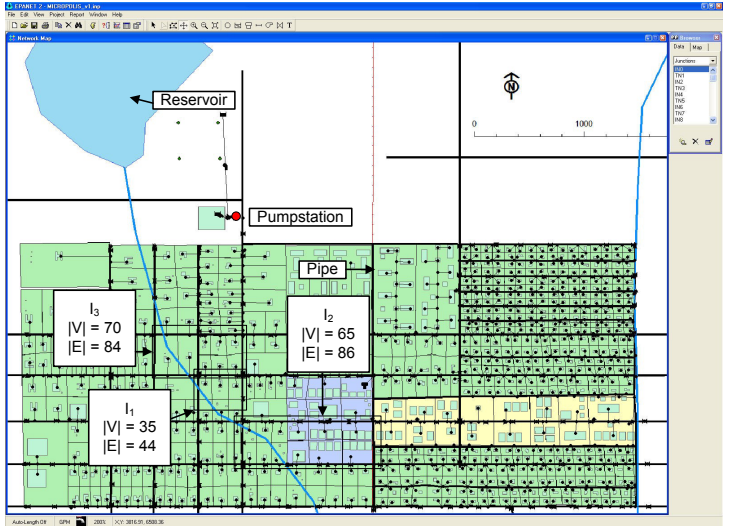

Figure 2: Micropolis [38] virtual city model showing the zones of interest for validation

The sensor and beacon placement formulation determined in the same formulation (joint formulation) is solved using a mixed integer nonlinear solver MINLP [39], which implements a Branch and Bound $(\operatorname{minlpBB})$ algorithm. Two separate solutions, denoted as greedy, and linear, are implemented in $A M P L$ [41]. The sensor deployment algorithm aims to minimize the efneste in greedy and to minimize $u b f n$ in linear. All formulations are solved by a MINLP solver, an online service for solving numerical optimization problems [42] [43] [44]. The MINLP solver is capable of solving a wide range of linear and nonlinear optimization problems. We divide the evaluation into three parts: (i) evaluation on the Net1 example; (ii) evaluation on the Micropolis example; (iii) evaluation of sensing coverage solutions for cases where localization is not required. We perform evaluations on the Net1 example to verify the accuracy and robustness of our models and algorithms. Micropolis evaluations are done to ensure the scalability of our algorithms to bigger networks. The evaluation of sensing coverage solutions is done to compare our algorithms with the state of the art. We note here that to the best of our knowledge, there is no current work in localizing leak/backflow events using static access points in the manner in which it is used in this paper. Models are run on a desk- 


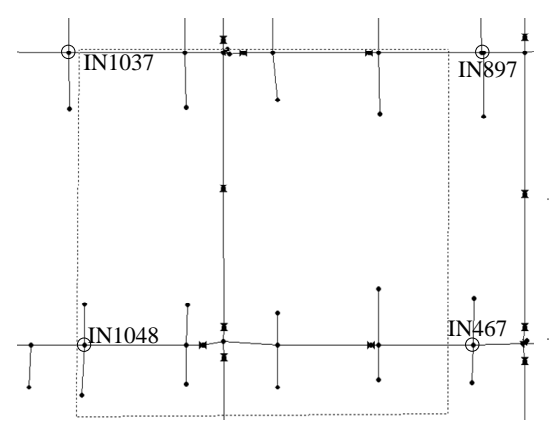

(a)

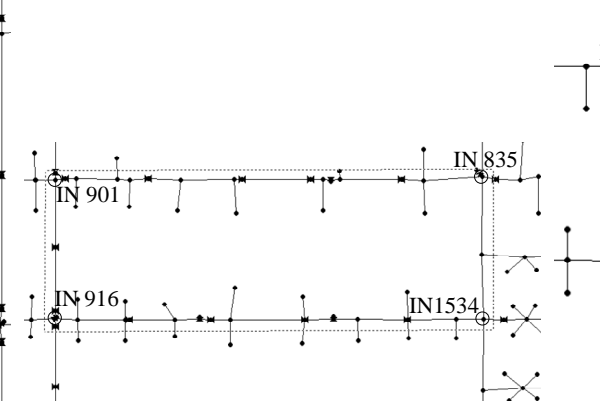

(b)

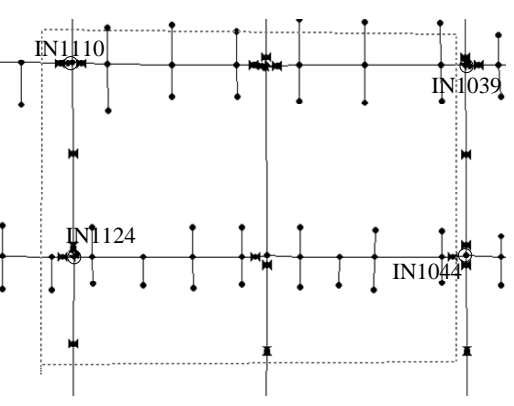

(c)

Figure 3: Zoomed in views of (a) Zone $I_{1}$, (b) Zone $I_{2}$, (c) Zone $I_{3}$

top with 3.7GiB Ram and Intel Core 2 Duo CPU E8400@3.00GHz $\times 2$.

The input parameters of our algorithms are the price of devices and cost budget. To simplify the calculations, these parameters are expressed as integer multiples of the price of sensors and beacons. We assume the price of a beacon to be an integer (say, c) times the price of of a sensor. A sensor's price is normalized to be 1 , thereby making the price of a beacon $c$. Throughout this evaluation, unless otherwise mentioned, the price of a beacon is 3 . The total cost budget varies from 10 to 70 units with an interval of 10 . When generating the data file for each zone in Micropolis example, we use the flow rates generated at hour 7 of the EPANET simulation, and eliminate edges whose flow probability is less than $1 \%$.

In order to compare the performance of an algorithm on different networks, we use the normalized sle instead of the absolute sle we defined before. Normalized sle is calculated by dividing absolute sle over the sum of leak probabilities of all edges. We denote normalized sle as normalized sle in subsequent discussion. The same applies to ale. The normalization is done so as to make the metric uniform among all network sizes.

When comparing our separate solutions to the joint solution, the cost budget is an input parameter. The metrics we use are the normalized sle, sensing coverage, detection rate, and normalized ale. For the Net1 example, we ran simulations with leaks placed on all edges, one at a time. For the Micropolis example, we run simulations on a group of edges (covering more than $75 \%$ edges) for each zone. For each run, we set an event on an edge and measure the detection rate, sle and other metrics among the group of edges. Each point is the
78750 average of 30 runs.

${ }_{78851}$ For the Net1 example, we also run sensitivity analysis by varying the leak distribution, sensorbeacon cost ratio, and number of simulation runs. We also check robustness of the formulations by adding restrictions on sensor and beacon placement locations.

We finally address the issue of maximizing sensing coverage in a given zone of interest with mobile sensors, since it is an essential prerequisite for locating leaks/backflows. It is also important when the utility manager is interested only in determining the presence, not the location of the leak/backflow. As mentioned before, the problem of ensuring a certain $L B S C$ while minimizing the number of sensors was formulated in [24] (Minimization of Number of Sensors, or MNS). In this paper, we compare our solution of MLBSC with MNS. The placement of sensors concerning the sensing coverage is determined using Flowsim for the MNS problem, CPLEX in MATLAB for the MLBSC problem, and a greedy heuristic implementation in MATLAB for the MASC problem.

For the three sensor deployment algorithms, the parameter that we varied is the number of sensors. For comparison, the metrics we use are: (i) average sensing coverage $(A S C)$; (ii) lower bound sensing coverage $(L B S C)$. We set the total number of sensors to 8 different values based on 8 degrees of coverage inputs to MNS $(0.2,0.3, \ldots 0.9)$ for the 3 different zones of interest in the Micropolis example.

\subsection{Results and Discussions}

\subsubsection{Net1 network results}

The results for the Net1 application are presented in Figures $4-8$. All the results are averaged over 


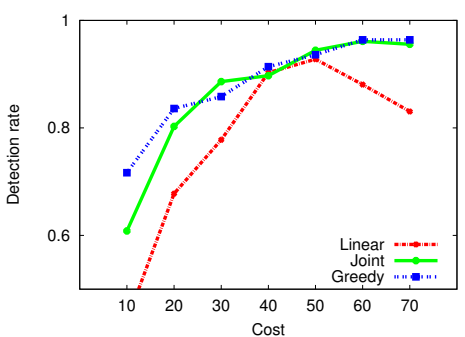

(a)

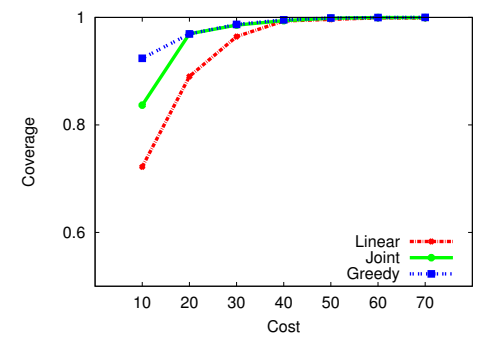

(b)

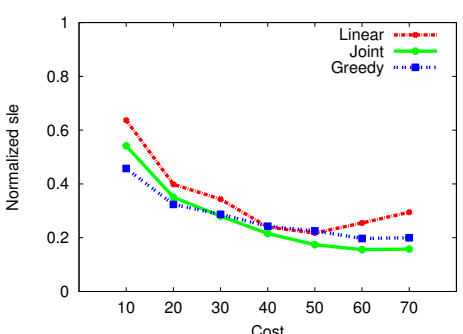

(d)

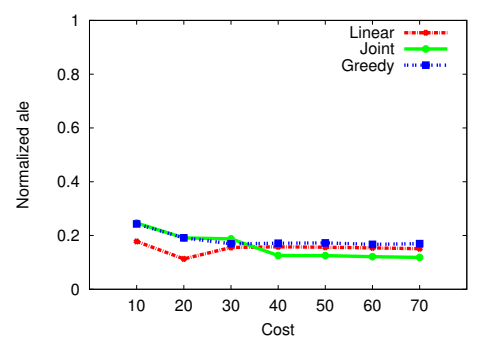

(c)

Figure 4: Model results for the Net1 example shown in Figure 1, with a uniform distribution of the leaks for (a) Detection rate, (b) Coverage, and (c) Average Localization Error, (d) System Localization Error

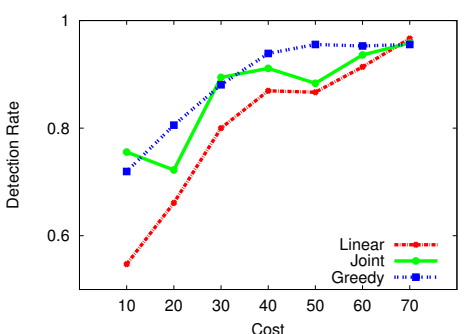

(a)

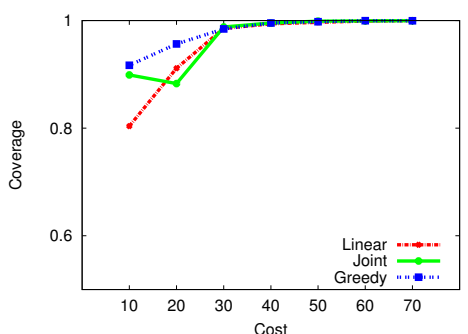

(b)

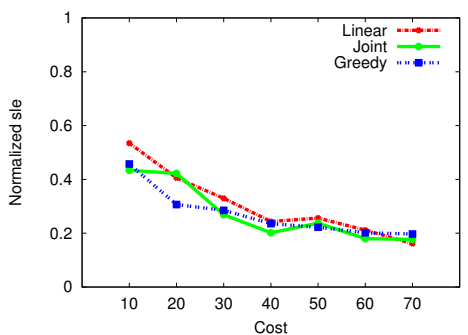

(d)

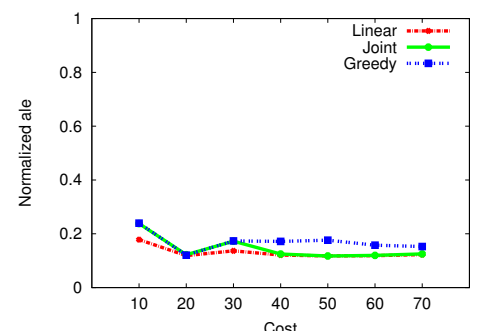

(c)

Figure 5: Model results for the Net1 example shown in Figure 1, with a random distribution of the leaks for (a) Detection rate, (b) Coverage, and (c) Average Localization Error, (d) System Localization Error

30 runs each, with leaks being present on each of ${ }_{82 \$ 23}$ each pipe has different leak probabilities). In both the pipes of the WDS.

${ }_{83824}$ cases, the cost ratio of a beacon to a sensor is 3 . As

831 the figures suggest, the general tendency is for the

In Figure 4, the distribution is uniform (i.e., the 825 coverage and detection rate to increase, whereas the sle and ale reduce. This is expected, since when more budget is available, it is possible to afford leak probability is the same in all edges), whereas in Figure 5, the leak distribution is random (i.e., sampled from a uniform random distribution where 


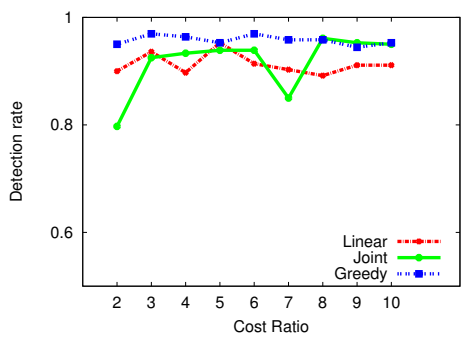

(a)

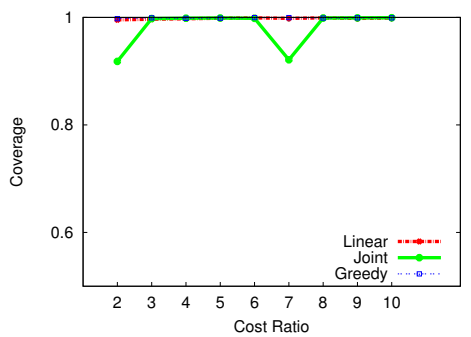

(b)

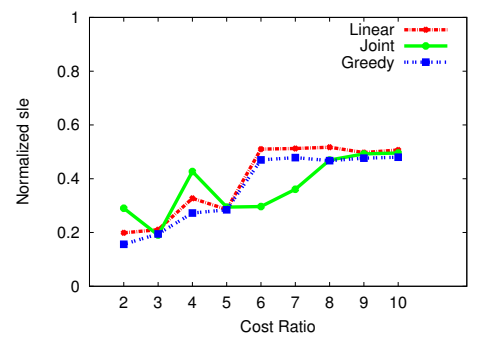

(c)

Figure 6: Model results for the Net1 example shown in Figure 1, while varying cost ratio between sensors and beacons for (a) Detection rate, (b) Coverage, and (c) System Localization Error

more devices to improve sle and ale, and that in turn improves the coverage and detection rate.

In Figure 4, we notice that the normalized sle for

Table 4: Scenarios for which sensor and beacon placements are restricted

\begin{tabular}{|l|l|l|}
\hline Scenario & $\begin{array}{l}\text { Beacon } \\
\text { restriction }\end{array}$ & $\begin{array}{l}\text { Sensor } \\
\text { restriction }\end{array}$ \\
\hline 1 & 12,21 & 12,22 \\
\hline 2 & $11,12,21$ & $11,12,22$ \\
\hline 3 & $11,12,21,31$ & $11,12,21,31$ \\
\hline 4 & $9,11,12,21,31$ & \\
\hline 5 & & $9,11,12,21,31$ \\
\hline 6 & $9,11,12,21,31$ & $9,11,12,21,31$ \\
\hline
\end{tabular}

Figure 6 illustrates the sensitivity of performance metrics to the beacon-sensor cost ratio. The cost budget is set to be 50 . Normalized sle increases overall with increasing the cost ratio. This is because fewer beacons are placed. Fewer beacons lead to an increased ale, because there are more pipes between each of the beacon pairs. In case of a cost ratio of 2 , the joint formulation places beacons at all vertices, thereby spending less on sensors, which is determined to be the optimal for that cost ratio. This leads to a higher coverage, and a lower sle for the cost ratio of 3 . However, the greedy formula-
835 tion is able to maintain an increasing trend in sle 836 because by nature, it optimizes the average cover${ }_{837}$ age and localization errors. Above a cost ratio of 5, the trend is more uniform over all formulations.

We test robustness of our solution by adding constraints to the placement of beacons and sensors, as shown in Figure 7. The nodes that are restricted are as described in Table 4 . The cost budget is set to be 50 .

Restricting the placement of beacons and sensors harms the coverage and detection rate as expected. But mainly, with a higher cost, the beacon placement restriction affects sle more than the sensor placement restriction. It is observed that when both sensor and beacon placement are restricted severely (Scenario 6), the beacons are deployed at all possible vertices, even though it reduces the number of sensors slightly as compared to a case when there are fewer restrictions (Scenario 3).

The obtained coverage, detection rate, and sle values for varying number of runs, are shown in Figure 8 . The cost budget is set to 50 . However, the error bars for coverage gradually reduce. In case of the normalized sle, the error bars are wide because the edge localization errors are different for each edge. Moreover, if the leak is not detected, the normalized sle is 1 , thereby increasing the variance in the results. 851 852 853 854 855 856 857 858 859 860
Table 5: Sensitivity Analysis Summary

\begin{tabular}{|l|l|l|l|}
\hline Parameter & sle & ale & AchSC \\
\hline Total budget (cost) & $\downarrow$ & $\downarrow$ & $\uparrow$ \\
\hline Cost ratio $\left(\frac{b p}{s p}\right)$ & $\uparrow$ & $\uparrow$ & $\sim$ \\
\hline $\begin{array}{l}\text { Number of restricted } \\
\text { vertices for beacons }\end{array}$ & $\uparrow$ & $\uparrow$ & $\uparrow$ \\
\hline $\begin{array}{l}\text { Number of restricted } \\
\text { vertices for sensors }\end{array}$ & $\uparrow$ & $\downarrow$ & $\downarrow$ \\
\hline
\end{tabular}




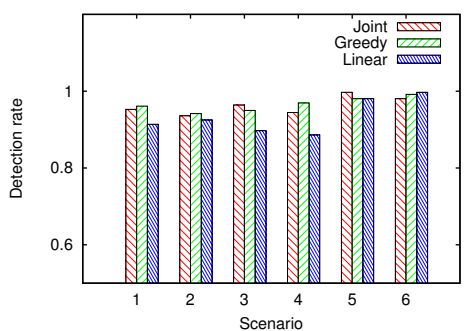

(a)

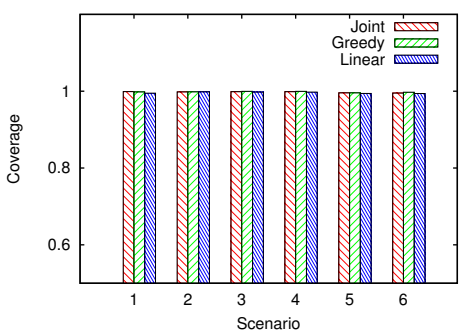

(b)

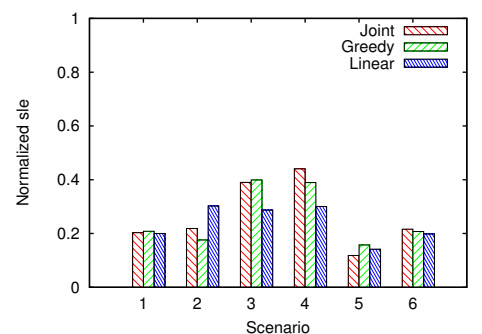

(c)

Figure 7: Model results for the Net1 example shown in Figure 1, when location of beacons and sensors may be restricted for (a) Detection rate, (b) Coverage, and (c) System Localization Error

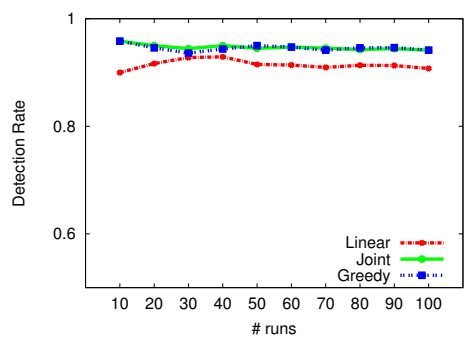

(a)

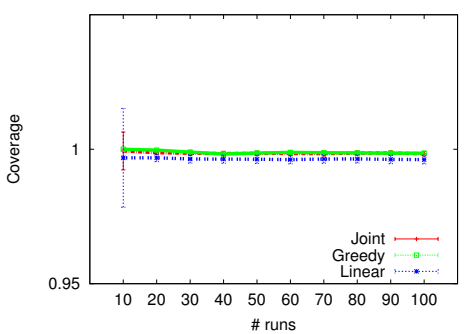

(b)

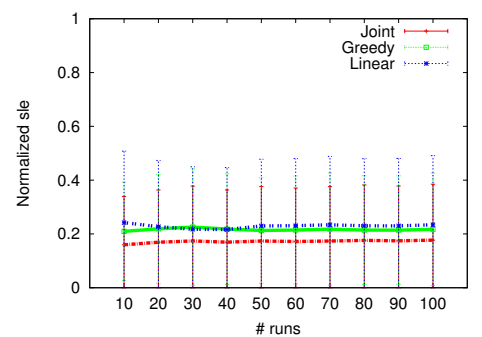

(c)

Figure 8: Model results for the Net1 example shown in Figure 1, for different number of simulation runs for (a) Detection rate, (b) Coverage, and (c) System Localization Error

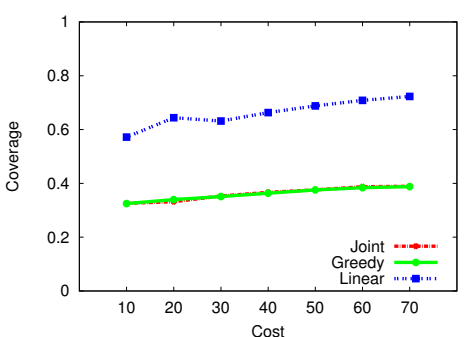

(a)

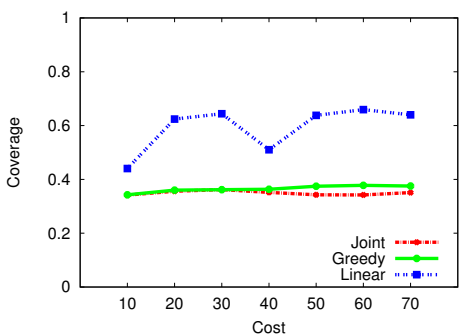

(b)

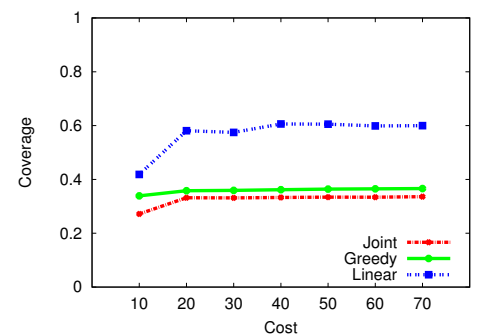

(c)

Figure 9: Comparison of average sensing coverage determined mathematically for (a) Zone $I_{1}$, (b) Zone $I_{2}$, (c) Zone $I_{3}$

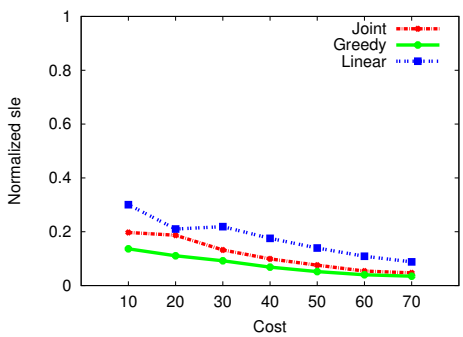

(a)

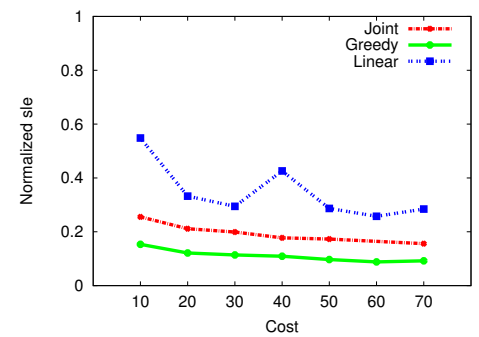

(b)

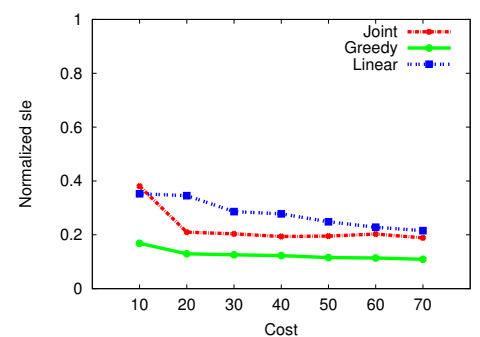

(c)

Figure 10: Comparison of normalized sle determined mathematically for (a) Zone $I_{1}$, (b) Zone $I_{2}$, (c) Zone $I_{3}$

The summary of our sensitivity analysis is described in Table 5. The table indicates the effect
889 of increasing the parameters in the first column 890 on the sle, ale, and AchSC. We recall here that 


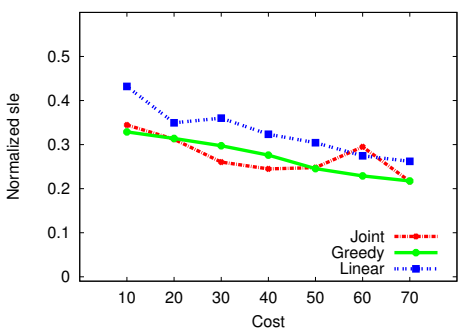

(a)

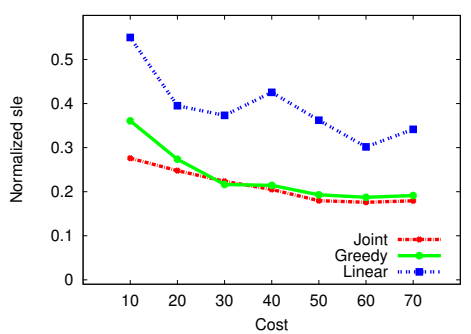

(b)

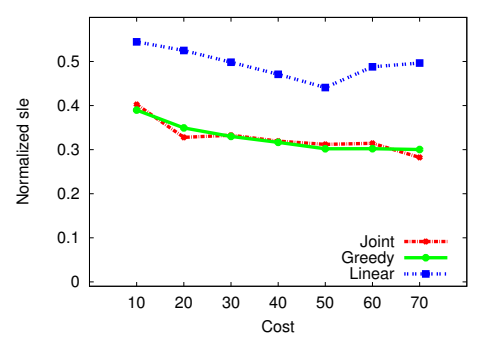

(c)

Figure 11: Comparison of achieved normalized sle in Flowsim for (a) Zone $I_{1}$, (b) Zone $I_{2}$, (c) Zone $I_{3}$

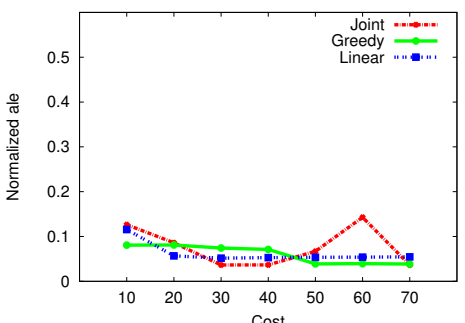

(a)

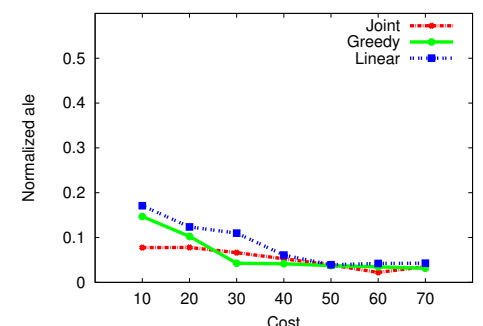

(b)

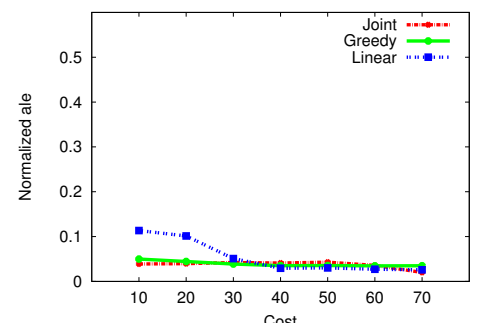

(c)

Figure 12: Comparison of average normalized ale for (a) Zone $I_{1}$, (b) Zone $I_{2}$, (c) Zone $I_{3}$

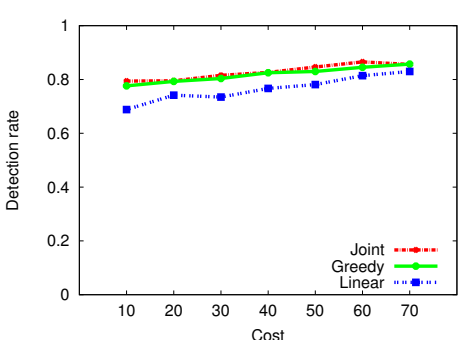

(a)

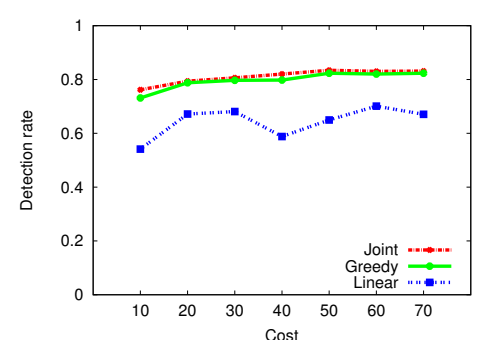

(b)

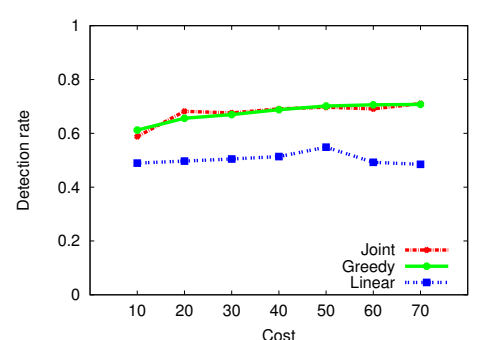

(c)

Figure 13: Comparison of detection ratio in Flowsim for (a) Zone $I_{1}$, (b) Zone $I_{2}$, (c) Zone $I_{3}$

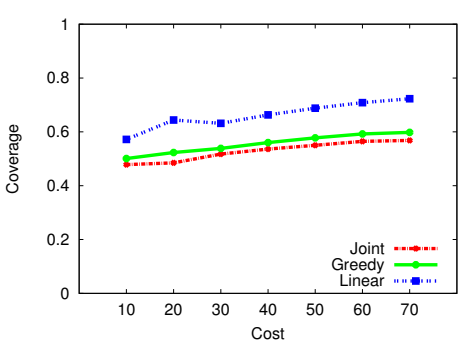

(a)

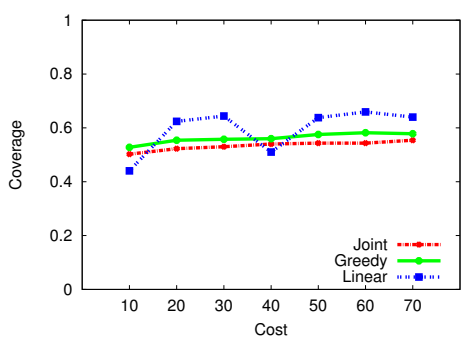

(b)

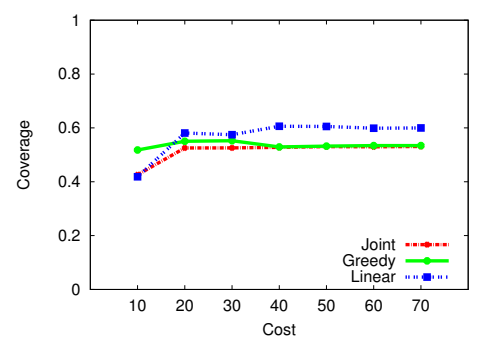

(c)

Figure 14: Comparison of coverage in Flowsim for (a) Zone $I_{1}$, (b) Zone $I_{2}$, (c) Zone $I_{3}$

sle $=$ AchSC $\cdot$ ale $+(1-A c h S C) \sum_{e_{k h}} l_{k h}$. The sym- 89893 that the metric decreases when the corresponding bol $\uparrow$ indicates that the metric increases when the ${ }_{89894}$ parameter is increased, and $\sim$ indicates that in- 
on the metric.

\subsubsection{Comparison of joint and disjoint approaches} in the Micropolis network

Once the sensor and beacon placements (i.e., $\mathbf{S}$ and $\mathbf{B}$ ) are obtained from the MINLP solver, we plug in the values in the expression for sle from Equation 2 to obtain a theoretical value of the sle using the expression of efneste from Equation 10 to obtain the theoretically expected sensing coverage. Figures 9 and 10 indicate average sensing coverage and normalized sle obtained mathematically for each of the three zones of interest in Micropolis network.

As Figure 10 shows, the sle reduces when more budget is available. This is expected in all the formulations because the objective in the joint formulation is to minimize ale, and ale is directly related to sle. The term sle only places more emphasis on coverage as compared to ale. Minimizing only ale can also have a similar effect on sle. In case of the linear formulation, we select the best distribution of cost between sensors and beacons at each step of the exhaustive search. However, sle decreases with increasing budget except in a very few outlier cases (only 1 in this case). This may be attributed to the difference in where the same number of sensors are placed as compared to the case with lower cost. As Figure 9 shows, the coverage at a cost of 40 units in Zone 2 is lower than that for cost of 30 . This is possible because the linear formulation maximizes the minimum edge coverage, thereby placing more emphasis on those edges with smaller flows and leak probability. Also, some of the extra cost is spent on placing more beacons. The lower coverage has a direct impact, therefore, on sle. However, as the figures indicate, such variations are more of an outlier than the norm.

Figures 11 - 14 indicate that results obtained through simulations match the mathematically determined values. Figures 11 and 12 indicate that normalized sle decreases with increasing the available budget as expected, except for one case of Zone 1 when the cost is 60 units. Zone 1 has a relatively higher uneven distribution of flows at the junctions. For a cost of 60 , there are only 11 beacons placed as opposed to 16 beacons placed when a 50 unit budget is available. Theoretically, from Figure 10, the sle has to reduce. However, the budget put into increasing the coverage, so as to improve sle, has a smaller impact than the number of beacons might have had, as can be seen in Figures 14 and 13.
This is because the flow distribution at junctions in Zone 1 has more variance than in Zones 2 or 3 , and the increase in number of sensors is not sufficient to cover pipes with low flow rates. The increased cost invested in sensor placement is not sufficient to reduce sle as much as expected. This is also more of an outlier than the norm.

In general we notice that all three formulations present an increasing trend in coverage and detection rate and a decreasing trend in sle and ale, which is expected. The greedy performs nearly as good as and sometimes better than the joint formulation although the joint formulation is more rigorous. The linear formulation is the computationally fastest of the three methods. However, it comes at a cost of not being as accurate or effective as the others. In simulations as well, greedy and joint solutions perform very well. Additionally, the greedy solution has consistent results with no outliers.

\subsubsection{Comparison of Sensor Deployment Algo- rithms to MNS [24]}

As we have discussed in Section 4.2, when we are interested only in identifying the presence of a leak/backflow and not its location, the MASC problem may be solved using a greedy algorithm, or the MLBSC problem may be solved using a linear formulation. The cost budget for the sensors is fixed based on the outputs of the MNS solution because the objective of MNS is minimizing the number of sensors whereas number of sensors is a constraint in MASC and MLBSC.

The $A S C$ and $L B S C$ are calculated mathematically by plugging in the sensor deployment configuration in their respective expressions using MATLAB. The results are plotted in Figures 15 and 17. The $A S C$ and $L B S C$ achieved in simulation using FlowSim is presented in Figure 16 and Figure 18. Here, each scenario is simulated 100 times, and the figures plot the mean and one standard deviation (if applicable).

As the number of sensors increases, the achieved $A S C$ uniformly increases for both MASC and MNS problems in both simulation and analytically, as shown in Figure 16 and Figure 15. It is interesting to observe in Figure 16 that the achieved $A S C$ with the MASC is usually higher than that of MNS, except in one case (Zone $I_{2}$, number of sensors is 1328). This is because the algorithm in MASC is a greedy heuristic and does not always achieve the optimal solution. However, in most cases, MASC performs better than MNS. Also, increasing the num- 


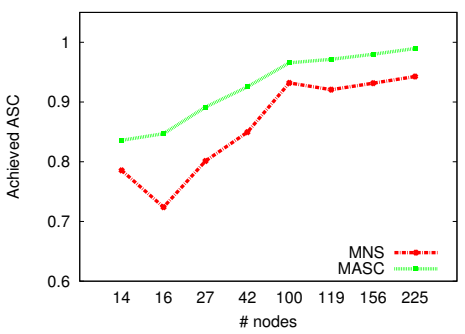

(a)

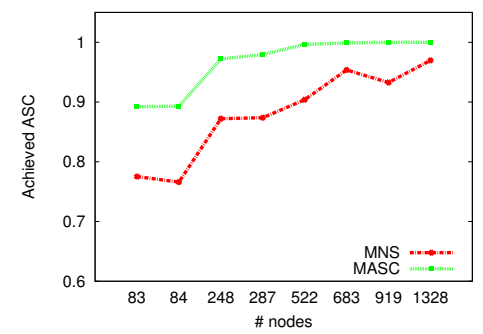

(b)

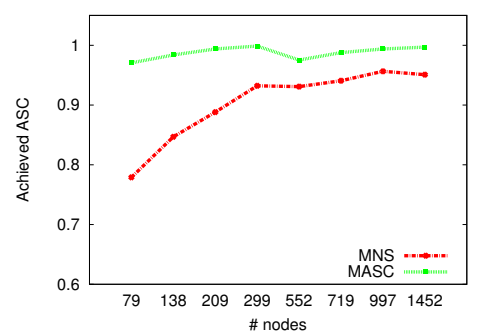

(c)

Figure 15: Comparison between MNS and MASC for achieved average sensing coverage in MATLAB for (a) Zone $I_{1}$, (b) Zone $I_{2}$, (c) Zone $I_{3}$

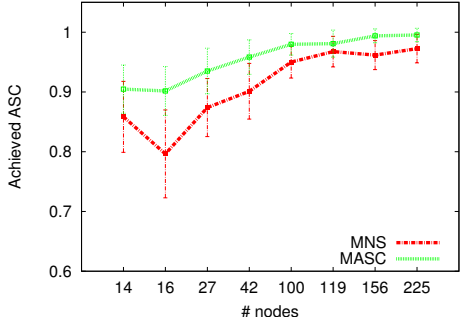

(a)

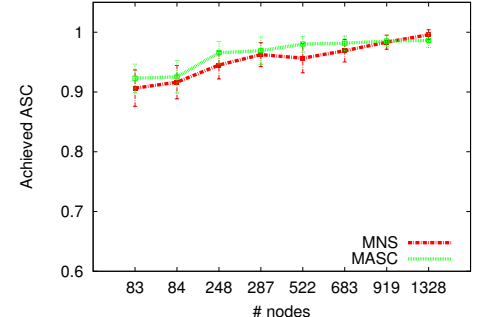

(b)

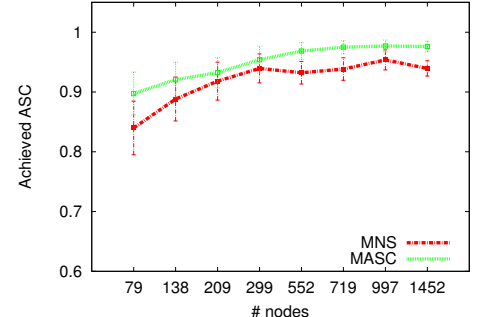

(c)

Figure 16: Comparison between MNS and MASC for achieved average sensing coverage over 100 runs in FlowSim for (a) Zone $I_{1}$, (b) Zone $I_{2}$, (c) Zone $I_{3}$

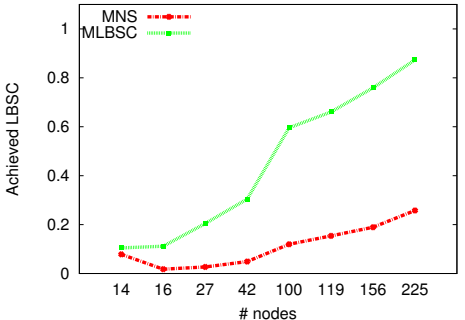

(a)

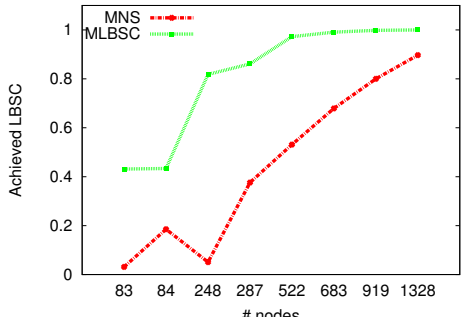

(b)

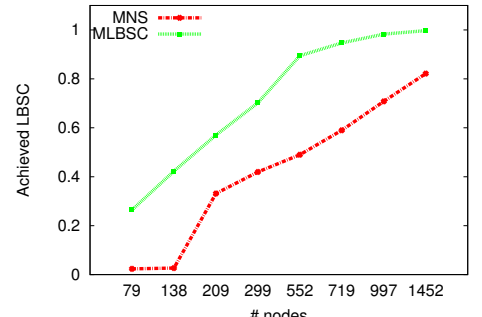

(c)

Figure 17: Comparison between MNS and MLBSC for achieved lower bound sensing coverage in MATLAB (a) Zone $I_{1}$, (b) Zone $I_{2}$, (c) Zone $I_{3}$

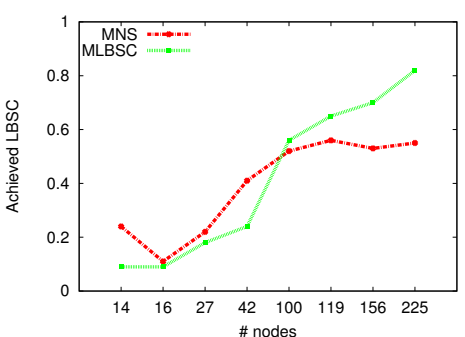

(a)

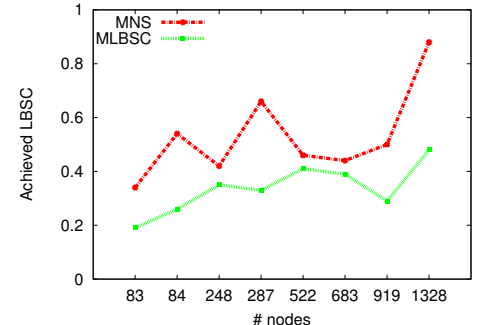

(b)

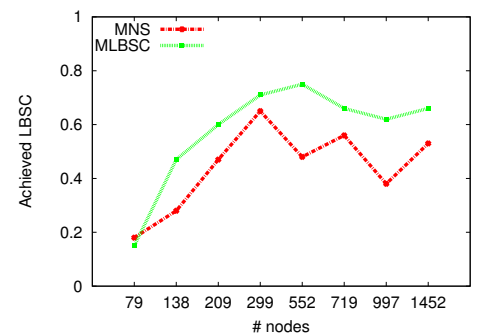

(c)

Figure 18: Comparison between MNS and MLBSC for achieved lower bound sensing coverage over 100 runs in FlowSim for (a) Zone $I_{1}$, (b) Zone $I_{2}$, (c) Zone $I_{3}$ 
ber of sensors does not increase $L B S C$ uniformly in MNS for all cases. This is because number of sensors is not a constraint, but the optimization function in MNS. In MNS, for a given $L B S C$, the number of sensors is obtained using a greedy heuristic. Whereas in MASC, a cost budget is given and the sensor placement to maximize $L B S C$ is then obtained.

It is interesting to observe in Figure 18 that as the number of sensors is increased, the achieved $L B S C$ does not show any discernable trend in simulations. However, the $L B S C$ shows an increasing trend in theory, as shown in Figure 17. This is because of the random movement of sensors at junctions. The randomness has a lower effect on an average in the case of $A S C$, but when movement of sensors through individual edges is considered in calculating the $L B S C$, the results become less predictable. In the analytical results in Figure 17, it is clear that $L B S C$ from MNS is lower than that of MLBSC, since the MLBSC problem is designed to maximize $L B S C$, and we obtain the optimal solution by solving MLBSC. We have obtained the results in Figure 18 for 100 runs in FlowSim. Increasing the number of runs may have an impact on the $L B S C$ achieved to some extent due to the randomness in sensor movement (i.e., edges with lower flows may be covered with a higher degree if the number of runs is high enough). However, in a real leak/backflow detection exercise, we may be deploying the sensors only a limited number of times.

\section{Conclusions}

This paper addresses the detection of leak/backflow in WDSs. We present a mathematical model for the optimization problem minimizing the system localization error in WDSs subject to a budget constraint. The problem of determining the placement of sensors and beacons in a single joint formulation is a mixed integer nonlinear optimization problem. To overcome the time complexity of the joint formulation, we also propose two disjoint schemes: a greedy solution that combines the Minimization of the Expected False Negative RateSystem Loealization Error (MEFNMESLE) and Minimization of the Linearized Average Localization Error (MLALE) problems; a linear programming solution that combines the Minimization of the Upper Bound ${ }_{1095}$ False Negative RateEdge Localization Error
(MUBFNMUBELE) and Minimization of the ${ }^{1051}$ Linearized Upper Bound Edge Localization Er- ${ }_{1052}$ ror (MLUBLE) problems. We have performed ${ }_{1053}$ extensive evaluations on a small network (i.e., ${ }^{1054}$ EPANET example Net1), as well as three parts 1055 of the Micropolis virtual city. Compared to joint, ${ }_{1056}$ separate methods greedy and linear achieve ${ }^{1057}$ a much lower time complexity by linearizing ${ }_{1058}$ the beacon placement formulation. When we 1059 are interested only in detecting the presence of 1060 the leak/backflow, we propose sensor placement ${ }_{1061}$ mechanisms to Maximize the Average Sensing ${ }_{1062}$ Coverage (MASC) and to Maximize the Lower ${ }_{1063}$ Bound Sensing Coverage (MLBSC). Both MASC ${ }_{1064}$ and MLBSC outperform the state of the art 1065 solution that Minimizes the Number of Sensors ${ }_{1066}$ (MNS). Therefore, the key conclusion from our ${ }^{1067}$ experiments is that the disjoint algorithm greedy ${ }_{1068}$ is an efficient and reliable algorithm for sensor and 1069 beacon placement problem in WDSs. We leave ${ }_{1070}$ for future work the extension of our algorithms to ${ }_{1071}$ WDSs where the flow rate in the pipes varies over ${ }_{1072}$ time.

\section{Acknowledgements}

1074

This material is based upon work supported by 1075 the National Science Foundation (NSF) under grant ${ }_{1076}$ number 1253968 and partially by the United States - 1077 Binational Science Foundation (BSF) under project ${ }_{1078}$ number 2012112.

\section{Appendix A. Proof of Approximation Ratio 1080}

The approximation ratio of the greedy algorithm 1081 is $\left(1+\frac{1}{e-1}\right)$. Before the proof, we define four opera- ${ }_{1082}$ tions " $\cap "$, " $\cup ", "+"$, "-", and one relation " $\in$ " on 1083 vectors:

$$
\begin{aligned}
& \mathbf{A} \cap \mathbf{B}=\left[\min \left\{a_{1}, b_{1}\right\}, \ldots, \min \left\{a_{n}, b_{n}\right\}\right] \\
& \mathbf{A} \cup \mathbf{B}=\left[\max \left\{a_{1}, b_{1}\right\}, \ldots, \max \left\{a_{n}, b_{n}\right\}\right] \\
& \mathbf{A}+\mathbf{B}=\left[a_{1}+b_{1}, \ldots, a_{n}+b_{n}\right] \\
& \mathbf{A}-\mathbf{B}=\left[\max \left\{0, a_{1}-b_{1}\right\}, \ldots, \max \left\{0, a_{n}-b_{n}\right\}\right]
\end{aligned}
$$

where $\mathbf{X} \in \mathbf{A}$ for $a_{i} \geq x_{i}$ for $0 \leq i \leq n$, and $\mathbf{A} 1090$ and $\mathbf{B}$ are vectors in $n$-dimensional space, $\mathbf{X}$ is a 1091 base vector in $n$-dimensional space.

Proof: The objective function $f: \mathbf{S} \rightarrow \mathbf{R}$ is given ${ }^{-1092}$ by:

$$
f(\mathbf{S})=m-\sum_{j=1}^{m} \prod_{i=1}^{n} p_{i j}^{s_{i}}
$$




17

where $p_{i j}=\beta_{i j} \in[0,1]$ are constants, $\mathbf{S}=$ $\left[s_{1}, s_{2}, \ldots, s_{n}\right]$ is a vector and $s_{i}$ specifies the number of sensors inserted at vertex $v_{i}$. The vectors in the standard basis for $n$-dimensional space comprise the basic elements in $\mathbf{S}$.

Let $\mathbf{S}_{k}$ denote the first $k$ elements selected by the greedy algorithm and let $\mathbf{S}^{*}$ denote the actual optimum, $f\left(\mathbf{S}^{*}\right)=O P T$. The greedy algorithm will select exactly $c$ elements, i.e. $\mathbf{S}_{c}$ is the vector returned by the algorithm. We claim by induction that the inequation A.1 holds for $0 \leq k \leq c$, where $c$ is the given number of sensors.

$$
f\left(\mathbf{S}^{*}\right)-f\left(\mathbf{S}_{k}\right) \leq\left(1-\frac{1}{c}\right)^{k} f\left(\mathbf{S}^{*}\right)
$$

The base case $k=0: f\left(\mathbf{S}^{*}\right)-f([0, \ldots, 0]) \leq f\left(\mathbf{S}^{*}\right)$ holds.

Suppose the inequation A.1 holds true for the $k^{t h}$ step. We now prove it also holds for the $(k+1)^{t h}$ step. First, we prove three basic inequations:

$$
f(\mathbf{A} \cup \mathbf{B}) \geq f(\mathbf{A}) \quad \text { or } \quad f(\mathbf{B})
$$

\section{Proof:}

$$
f(\mathbf{A} \cup \mathbf{B})
$$

Proof: Please refer to the proof of inequation A.2.

$$
f(\mathbf{A}+\mathbf{B}) \leq f(\mathbf{A})+f(\mathbf{B})
$$

\section{Proof:}

$$
\begin{aligned}
& f(\mathbf{A}+\mathbf{B}) \\
& =m-\sum_{j=1}^{m} \prod_{i=1}^{n} p_{i j}^{a_{i}+b_{i}} \\
& =\sum_{j=1}^{m}\left(1-\prod_{i=1}^{n} p_{i j}^{a_{i}} * p_{i j}^{b_{i}}\right) \\
& =\sum_{j=1}^{m}\left(1-\prod_{i=1}^{n} p_{i j}^{a_{i}} * p_{i j}^{b_{i}}+\prod_{i=1}^{n} p_{i j}^{a_{i}}-\prod_{i=1}^{n} p_{i j}^{a_{i}}\right)
\end{aligned}
$$

$$
\begin{aligned}
& =\sum_{j=1}^{m}\left(1-\prod_{i=1}^{n} p_{i j}^{a_{i}}+\prod_{i=1}^{n} p_{i j}^{a_{i}} *\left(1-\prod_{i=1}^{n} p_{i j}^{b_{i}}\right)\right. \\
& \leq \sum_{j=1}^{m}\left(1-\prod_{i=1}^{n} p_{i j}^{a_{i}}+1-\prod_{i=1}^{n} p_{i j}^{b_{i}}\right) \\
& =2 m-\sum_{j=1}^{m} \prod_{i=1}^{n} p_{i j}^{a_{i}}-\sum_{j=1}^{m} \prod_{i=1}^{n} p_{i j}^{b_{i}} \\
& =f(\mathbf{A})+f(\mathbf{B})
\end{aligned}
$$

We define the marginal value of element $E$ with ${ }^{1135}$ respect to $\mathbf{S}$ as $f_{\mathbf{S}}(\mathbf{X})=f(\mathbf{S}+\mathbf{X})-f(\mathbf{S})$. Now we ${ }_{1136}$ can prove inequation A.5:

$$
f(\mathbf{A})-f(\mathbf{B}) \leq \sum_{\mathbf{X} \in \mathbf{A}-\mathbf{B}} f_{\mathbf{B}}(\mathbf{X})
$$

$$
\begin{aligned}
& f(\mathbf{A})-f(\mathbf{B}) \\
& \leq f(\mathbf{A} \cup \mathbf{B})-f(\mathbf{B}) \\
& \leq f(\mathbf{B})+f(\mathbf{A}-\mathbf{B})-f(\mathbf{B}) \\
& \leq \sum_{\mathbf{X} \in \mathbf{A}-\mathbf{B}} f_{\mathbf{B}}(\mathbf{X})
\end{aligned}
$$
1144

According to the greedy Algorithm 2, the ele- ${ }_{1145}$ ment $\mathbf{X}_{k+1}$ selected in the $(k+1)^{t h}$ step maximizes $\quad 1146$ $f_{\mathbf{S}_{k}}\left(\mathbf{X}_{k+1}\right)$ among the remaining elements, includ- ${ }_{1147}$ ing all the elements in $\mathbf{S}^{*}-\mathbf{S}_{k}$. This implies that ${ }_{1148}$ the element $\mathbf{X}_{k+1}$ has the marginal value:

$$
\begin{array}{rlr}
f_{\mathbf{S}_{k}}\left(\mathbf{X}_{k+1}\right) & \geq \frac{1}{\left|\mathbf{S}^{*}-\mathbf{S}_{k}\right|} \sum_{\mathbf{X} \in \mathbf{S}^{*}-\mathbf{S}_{k}} f_{\mathbf{S}_{k}}(\mathbf{X}) \\
& \geq \frac{1}{c}\left(f\left(\mathbf{S}^{*}\right)-f\left(\mathbf{S}_{k}\right)\right)
\end{array}
$$
Finally, we can prove inequation A.1 at the $k+1{ }_{1153}$ step:

$$
\begin{aligned}
& f\left(\mathbf{S}^{*}\right)-f\left(\mathbf{S}_{k+1}\right) \\
& =f\left(\mathbf{S}^{*}\right)-f\left(\mathbf{S}_{k}\right)-f_{\mathbf{S}_{k}}\left(\mathbf{X}_{k+1}\right) \\
& \leq f\left(\mathbf{S}^{*}\right)-f\left(\mathbf{S}_{k}\right)-\frac{1}{c}\left(f\left(\mathbf{S}^{*}\right)-f\left(\mathbf{S}_{k}\right)\right) \\
& =\left(1-\frac{1}{c}\right)\left(f\left(\mathbf{S}^{*}\right)-f\left(\mathbf{S}_{k}\right)\right) \\
& \leq\left(1-\frac{1}{c}\right)^{k+1} f\left(\mathbf{S}^{*}\right)
\end{aligned}
$$
1154 1155 1156

Using the claim for $k=c$, we get 
then we have approximation ratio:

$$
r=\frac{f\left(\mathbf{S}^{*}\right)}{f\left(\mathbf{S}_{i}\right)} \leq 1+\frac{1}{e-1}
$$

\section{References}

[1] C. Copeland, M. Tiemann, Water infrastructure needs and investment: review and analysis of key issues, Tech. rep., US Congressional Research Service (Report RL31116) (2010).

[2] U.S Environmental Protection Agency, Aging water infrastructure research, http://www.epa.gov/awi/, [Online; accessed 30-Oct-2013].

[3] American Water Works Association, Buried no longer: confronting america's water infrastructure challenge, http://www . awwa.org/infrastructure, [Online; accessed 30-Oct-2013].

[4] U.S Environmental Protection Agency, Control and mitigation of drinking water losses in distribution systems, Publication No. EPA 816-D-09-001.

[5] M. Mortazavi-Naeini, G. Kuczera, A. S. Kiem, L. Cui, B. Henley, B. Berghout, E. Turner, Robust optimization to secure urban bulk water supply against extreme drought and uncertain climate change, Environmental Modelling \& Software.

[6] J. Thornton, R. Sturm, G. Kunkel, Water loss control, 2nd ed., New York: McGraw-Hil.

[7] J. Meseguer, J. M. Mirats-Tur, G. Cembrano, V. Puig, J. Quevedo, R. Prez, G. Sanz, D. Ibarra, A decision support system for on-line leakage localization, Environmental Modelling \& Software.

[8] Pure Technologies, Sahara leak \& gas pocket detection, http://www.puretechltd.com/products/sahara/ sahara_leak_gas_pocket.shtml, [Online; accessed 30Oct-2013].

[9] Pure Technologies, Smartball for water and wastewater water mains, http://www.puretechltd.com/products/ smartball/smartball_leak_detection.shtml, [Online; accessed 30-Oct-2013].

10] Pure Technologies, Pipediver, http://www. puretechltd.com/products/pipediver/pipediverpccp.shtml, [Online; accessed 30-Nov-2013].

[11] T. Lai, Y. Chen, P. Huang, H. Chu, Pipeprobe: a mobile sensor droplet for mapping hidden pipeline, in: SenSys, 2010.

[12] D. Trinchero, R. Stefanelli, Microwave architectures for wireless mobile monitoring networks inside water distribution conduits, Microwave Theory and Techniques, IEEE Transactions on

[13] D. M. Chatzigeorgiou, Analysis and design of an in-pipe system for water leak detection, Massachusetts Institute of Technology.

[14] M. K. Banks, A. Brovont, S. Pekarek, M. Porterfield, A. Salim, R. Wu, Development of mobile self-powered sensors for potable water distribution, http://cfpub.epa.gov/ncer_abstracts/index.cfm/ fuseaction/display.abstractDetail/abstract/9458/ report/2012, [Online; accessed 30-Oct-2013].

[15] D. Chatzigeorgiou, Y. Wu, D. Wu, K. Youcef-Toumi, A new in-pipe leak detection system, Mechatronics Research Laboratory, MIT.

[16] L. Perelman, A. Ostfeld, Operation of remote mobile ${ }_{1221}^{1286}$ sensors for security of drinking water distribution systems, Water Research.

[17] W. R. Furnass, S. R. Mounce, J. B. Boxall, Linking distribution system water quality issues to possible causes via hydraulic pathways, Environmental Modelling \& Software.

18] A. Rasekh, K. Brumbelow, Drinking water distribution systems contamination management to reduce public health impacts and system service interruptions, Environmental Modelling \& Software.

[19] U.S Environmental Protection Agency, Potential contamination due to cross-connections and backflow and the associated health risks, Office of Ground Water and Drinking Water, Washington DC.

[20] American Water Works Association, Reinvesting in drinking water infrastructure, http://www.win-water. org/reports/infrastructure.pdf, [Online; accessed 30-Oct-2013].

[21] O. Hunaidi, A. Wang, M. Bracken, T. Gambino, C. Fricke, Acoustic methods for locating leaks in municipal water pipe networks, in: International Conference on Water Demand Management, 2004, pp. 1-14.

[22] R. Puust, Z. Kapelan, D. Savic, T. Koppel, A review of methods for leakage management in pipe networks, Urban Water Journal.

[23] M. Convertino, Y. Liu, H. Hwang, Optimal surveillance network design: a value of information model, Complex Adaptive Systems Modeling 2 (1)

[24] M. Suresh, R. Stoleru, E. Zechman, B. Shihada, On event detection and localization in acyclic flow networks, Systems, Man, and Cybernetics: Systems, IEEE Transactions on.

[25] M. Suresh, R. Stoleru, R. Denton, E. Zechman, B. Shihada, Towards optimal event detection and localization in acyclic flow networks, in: ICDCN, 2012.

[26] M. Suresh, W. Zhang, W. Gong, A. Rasekh, R. Stoleru, M. Banks, Towards optimal monitoring of flow-based systems using mobile wireless sensor networks, ACM Transactions on Sensor Networks.

[27] M. Suresh, L. Smith, A. Rasekh, R. Stoleru, M. Banks, B. Shihada, Mobile sensor networks for leak and backflow detection in water distribution systems, in: AINA, 2014.

[28] M. Suresh, U. Manohar, A. G R, R. Stoleru, M. Kumar M S, A cyber-physical system for continuous monitoring of water distribution systems, in: WiMob, 2014.

[29] Canadian commercial newswire, Pure technologies awarded multi year leak detection contract in qatar, http://www. newswire.ca/en/story/1369537/puretechnologies-awarded-multi-year-leak-detectioncontract-in-qatar, [Online; Published 9-June-2014].

[30] J. Kim, G. Sharma, N. Boudriga, S. Iyengar, SPAMMS: a sensor-based pipeline autonomous monitoring and maintenance system, in: COMSNETS, 2010.

[31] T. Lai, W. Chen, K. Li, P. Huang, H. Chu, TriopusNet: automating wireless sensor network deployment and replacement in pipeline monitoring, in: IPSN, 2012.

[32] Z. Sun, P. Wang, M. Vuran, M. Al-Rodhaan, A. AlDhelaan, I. Akyildiz, MISE-PIPE: Magnetic inductionbased wireless sensor networks for underground pipeline monitoring, Ad Hoc Netw.

[33] A. Yazdani, P. Jeffrey, Complex network analysis of water distribution systems, Arxiv preprint arXiv: 1104.0121

[34] X. Li, W. Yu, X. Lin, S. Iyengar, On optimizing au-

\section{2}

1223 1224 1225 1226 1227 1228 1229 1230 1231 1232 1233 1234 1235 1236 1237 1238 1239 1240 1241 1242 1243 1244 1245 1246 1247 1248 1249 1250 1251 1252 1253 1254 1255 1256 1257 1258 1259 
tonomous pipeline inspection, Robotics, IEEE Transactions on.

[35] S. Xiong, L. Yu, H. Shen, C. Wang, W. Lu, Efficient algorithms for sensor deployment and routing in sensor networks for network-structured environment monitoring, in: INFOCOM, 2012.

[36] A. Rasekh, K. Brumbelow, Probabilistic analysis and optimization to characterize critical water distribution system contamination scenarios, Journal of Water Resources Planning and Management.

[37] Environmental Protection Agency, EPANET v2.0, Tech. rep. (2006).

[38] K. Brumbelow, J. Torres, S. Guikema, E. Bristow, L. Kanta, Virtual cities for water distribution and infrastructure system research, in: World Environmental and Water Resources Congress, 2007.

[39] R. Fletcher, S. Leyffer, Numerical experience with lower bounds for miqp branch-and-bound, in: SIAM J. Optimization, 1998, pp. 604-616.

[40] R. H. Byrd, J. Nocedal, R. A. Waltz, KNITRO: An Integrated Package for Nonlinear Optimization, in: LargeScale Nonlinear Optimization, 2006, pp. 35-59.

[41] R. Fourer, D. M. Gay, B. W. Kernighan, AMPL: A mathematical programming language, AT\&T Bell Laboratories Murray Hill, NJ 07974, 1987.

[42] J. Czyzyk, M. P. Mesnier, J. J. Moré, The neos server, IEEE Comput. Sci. Eng.

[43] E. D. Dolan, Neos server 4.0 administrative guide, arXiv preprint cs/0107034.

[44] W. Gropp, J. Moré, Optimization environments and the neos server. 